PROC. OF JSCE,

NO. 205, SEPT. 1972

\title{
EINE ERWEITERUNG DER THEORIE KLASSISCHER FLATTERNSCHWINGUNGEN
}

\author{
von Hiromichi HIGASHIHARA*
}

\section{ZUSAMMENF ASSUNG}

Theodorsensche Theorie über Schwingungen von Platten unter Wirkung des Windes, Theorie klassischer Flatternschwingungen, gibt dem Studium der aerodynamischen Stabilität von Schwingungen verschiedener Baustrukturen einen der wichtigsten theoretischen Gründe.

Diese Methode hat aber etwas theoretische Unbestimmtheit, die nicht kleine Schwierigkeit $z u$ verursachen scheint, wenn wir sie auf andere Schwingungen als die von Flügeln anwenden wollen. Denn die Theodorsensche Theorie kann nur eine spezielle Windgeschwindigkeit bestimmen, an der die Platte rein harmonisch oszilliert, und bringt keine Nachricht über andere Lagen. Diese Beschränkung wird oft tödlich, wenn die Windgeschwindigkeit nicht dafür $z u$ halten ist, unveränderlich $\mathrm{zu}$ bleiben.

Aber wir können die Theorie etwas erweitern und auch den Zustand von Schwingungen unter beliebiger Windgeschwindigkeit bestimmen, wenn wir die obige Unbestimmtheit genauer untersuchen und die Phänomene in einer allgemeineren und auch mathematisch exakteren Form feststellen.

\section{EINLEITUNG}

Dieses Studium enthält eigentlich zwei hauptsächliche Objekte, die zusammenfassend genannt werden können: Eine Erweiterung der Methode, die T. Theodorsen festgestellt hat, um die zweidimensionale Schwingung von Platten unter Wirkung des Windes $z u$ analysieren.

Dieses Studium verfolgt eine Art mathematische Tendenz in doppeltem Sinn, in positivem und auch in negativem. Was jenen betrifft, werden

* Lehrstuhl für Bautechnik, Abteilung der Zivilingenieurkunst, Technische Fakultät der Tokio Universität. einige theoretische Fragen der Theodorsenschen Theorie oder, man könnte anders sagen, der Theorie klassischer Flatternschwingung konsequent geprüft und abgeändert. Aber es scheint ja bemerkenswert, daß dieses theoretische Werk trotz seines abstrakten Aussehens eine sozusagen substantielle Erweiterung der vorhandenen Theorie hervorbringt, die nicht nur theoretisch sondern auch praktisch bedeutungsvoll sein kann.

Diese theoretische Prüfung bringt auch analytische Darstellung der Theodorsenschen Theorie, die für die Prüfung der Einwirkung der Störung der Luftströmung anwendbar ist, als Nebensprodukt hervor, wessen Erklärung mit Rücksicht auf den Raum leider nicht umhin kann, auf andere Gelegenheit aufzuschieben.

In Bezug auf den negativen Sinn, sind vorhandene Ergebnisse über die physikalische Bedeutung einiger Phänomene völlig so wie es ist angenommen worden, z.B. vor allem, die Bedingung von Kutta, die Strukturdämpfungskraft u.s.w., nämlich es ist vornehmlich beachtet worden, was für ein mathematischer Schluß aus einer physikalisch gleichen Hypothese hervorzubringen ist.

In diesem Referat werden hauptsächlich zwei Thesen geprüft:

(1) Ist das Ergebnis von Theodorsen denn einzig mögliche Schwingung unter der gegebenen Bedingung ?

(2) Wie ist der Zustand der Bewegung von Platten, wenn sie möglich ist, unter anderen Bedingungen als die von Theodorsen ?

Um sie zu lösen, stellen wir erstens die Theodorsensche Theorie mit analytischen Gleichungen explizite dar. Dann können diese Fragen ausschließlich analytisch gelöst werden, wobei die im Abschinitt I kurz eingeführten mathematischen Sätze wichtige Rolle spielen. Im Abschnitt II wird die Theodorsensche Theorie vom oben erwähnten Standpunkt geprüft, und die Fragen dieses Studiums werden festgestellt.

Die erste Hauptthese wird im tritten Abschnitt bejahend gelöst. Die Entwicklung von der ersten 
zur zweiten These wird im vierten Abschnitt mit Hilfe der Theorie von Funktionen komplexer Veränderlichen ausgeführt.

Im Gebiet der Aeronautik wurde die Aerodynamik des Flügels entwickelt von $\mathrm{H}$. Wagner, T. Theodorsen, H. G. Küssner, T. v. Kármán, W. R. Sears u.s. w., wo folgende zwei Probleme hauptsächlich untersucht wurden:

(1) klassische Flatternschwingung

(2) Windstoßproblem

$\mathrm{Da}$ (2) wesentlich vergänglich ist während (1) dauernd ist, könnte man sagen, daß sie voneinander ganz anders seien. Anderseits könnte man aber sagen, daß sie immer mit einer gemeinsamen Aufgabe $\mathrm{zu}$ tun hätten; nämlich die Bestimmung der aerodynamischen Kräfte. Das wäre selbstverständlich im Fall (2). Auch (1) ist keine Aushahme, denn die harmonische Schwingung wurde da nicht als "die Lösung der Bewegungsgleichungen" festgestellt. Diese Idee ist aber für die Baustrukturen sehr wichtig.

Lassen wir hier ein bißchen nachdenken über solch einen Unterschied der Schwerpunkte der Interesse der beiden Fachgebiete. $\mathrm{Da} B$ die Aeronautik die Bewegungsgleichungen verhältnismäßig vernachlässigt und nur die aerodynamischen Kräfte ernst nimmt, spiegelt die Umgebung der Flugzeuge richtig wieder und ist also vernünftig. Ihre Bewegung ist in Wirklichkeit ziemlich wohl kontrolliert, und ihre Bestimmung durch die Bewegungsgleichungen hat keine große Bedeutung. Hingegen im Fall der Baustrukturen, die eigentlich stillstehend und doch biegsam sind, wie zum Beispiel die lange Hängebrücke, ist die Bewegung selbst bedeutungsvoll und also die Bestimmung der Bewegung hat die Bedeutung erster Klasse.

Die Ergebnisse der Aeronautik können oft auch für die Bautechnik mächtige oder theoretisch führende Waffe sein; trotzdem ist die richtige Anwendung zu leisten nicht durch die zu direkte und rohe Einführung der Ergebnisse sondern dadurch, die Manier elastisch anzuwenden. Nämlich wenn der Gegenstand anders worden ist, ist die Theorie nur dadurch richtig anzuwenden, den Unterschied $z$ wischen der alten und der neuen Bedingungen ganau zu erkennen.

Auf jeden Fall wäre die obige Verschiedenheit der beiden Gesichtpunkte natürlich, und wenn nur dieses Elementarerkenntnis gehalten wird, so wäre der Unterschied zwischen den oben erwähnten Forschungen und diesem Studium deutlich; nämlich dieses legt seinen Schwerpunkt auf die Bewegung und nicht auf die aerodynamischen Kräfte. (Es wäre jetzt klar, warum der
Titel dieses Referats nicht "Eine Erweiterung der Theodorsenschen Theorie" sondern "... Flatternschwingungen " ist.)

Aber wenn es nur die rein harmonische Schwingung beträfe, wäre es doch keins mehr als eine Nachprüfung der Flatterntheorie. (Dieses Studium stellte theoretisch fest, keine besondere Schwingungsform voraussetzend, daß die Schwingung vom Theodorsenschen Typ die einzigartige Lösung unter der Flatternbedingung ausgenommen einen pathologischen Fall ist. Deshalb hat es einen ganz anderen Inhalt als die Flatterntheorie.) Aber es verfolgte ausführlich die wirklich realisierbare Bewegung und hat schließlich gegen beliebige Bedingung, die nicht immer die Flatternbedingung ist, die unter ihr entstehende Schwingung als die mathematisch exakte Lösung entscheidet können. Diese Erweiterung ist von einem klaren mathematischen Ausdruck begleitet.

Kármán und Sear entwickelten die Aerodynamik zuerst in einer allgemeineren Form und dann wandten sie auf die vorgenannten (1) und (2) an. Aber wie diese Autoren selbst äußerten, ist ihre Feststellung "wesentlich ein kurzer Weg" der Theodorsenschen Theorie und also keine Erweiterung im oben erwähnten Sinn des Autors. Auf diese Weise bestimmen die Theorien der obigen Forscher gegen gegebene Windgeschwindigkeit die da entstehende Schwingung nicht, was die Absicht dieses Studiums ist. Ihre Feststellungen hatten von Anfang an keine solche Absicht. Die Umstände sind ganz ähnlich $z$. B. für die Wagnersche Funktion.

Es ist aber immer wiederzuholen, da $\beta$ das keine Unehre dieser Theorien ist, sondern hat man vielmehr zu würdigen ihren vortrefflichen Sinn, den Charakter der Gegenstände der Aeronautik richtig vorauszunehmen. Im Gegenteil würde dieses Studium, das die Bestimmung der Bewegung zielt und beliebige Windgeschwindigkeit in seine Schußweite zu legen will, nicht zur Sache gehören vom Standpunkt der Aeronautik; trotzdem wenn man einmal die Aufmerksamkeit auf die Baustrukturen richtet, wie vorerwähnt, verändert die Situation sich vollständig und die Feststellung dieses Studiums erhält die raison d'être. Der auf die Baustrukturen einwirkende natürliche Wind verändert sich mannigfaltigerweise, und dieses Studium hat mit einem von verschiedenen Faktorenen seiner Mannigfaltigkeit, die Windgeschwindigkeit, $z u$ tun, und ist keins mehr. 


\section{MATHEMATISCHE VORBEREI- TUNGEN}

\section{(1) Konfluent hypergeometrische Funktionen}

Wenn $\alpha$ nicht ganze $Z$ ahl ist, so ist die normale Form der konfluent hypergeometrischen Funktionen erster Art folgenderweise definiert ${ }^{1)}$ :

$$
\begin{aligned}
G_{1}(\alpha, \gamma ; z)= & \frac{2}{1-e^{-2 \pi i \alpha}} \cdot \frac{\Gamma(\gamma)}{\Gamma(\alpha) \Gamma(\gamma-\alpha)} \\
& \times \int_{C_{1}} e^{z \zeta \zeta^{\alpha-1}(1-\zeta)^{\gamma-\alpha-1} d \zeta} \\
& \left(-\frac{3}{2} \pi<\arg . z<\frac{3}{2} \pi\right)
\end{aligned}
$$

Der Schleifenweg $C_{1}$ verläuft an den beiden Ufern einer geeigneten Halblinie und umkreist sodann den Nullpunkt im positiven Sinn.

Diese Funktion $G_{1}(\alpha, \gamma ; z)$ ist eindeutig und analytisch auf der ganzen Ebene der komplexen Zahlen außer dem originalen Punkt. Der unendlich ferne Punkt ist auch ihre singuläre Stelle, aber wir brauchen darüber nicht in Betracht $z u$ ziehen für unsere Frage.

Einige bestimmte Integrale, die sich in den folgenden Abschnitten zeigen, können mit den konfluent hypergeometrischen Funktionen dargestellt werden:

$$
\left.\begin{array}{l}
\int_{0}^{\infty} \sqrt{\frac{\zeta+1}{\zeta}} e^{-z \zeta} d \zeta=-\frac{\pi i}{4} G_{1}\left(\frac{1}{2}, 2 ; z\right) \\
\int_{0}^{\infty} \sqrt{\zeta(\zeta+1)} e^{-z \zeta} d \zeta=\frac{\pi i}{16} G_{1}\left(\frac{3}{2}, 3 ; z\right) \\
\int_{0}^{\infty} \zeta \sqrt{\zeta(\zeta+1)} e^{-z \zeta} d \zeta=-\frac{\pi i}{32} G_{1}\left(\frac{5}{2}, 4 ; z\right)
\end{array}\right\}
$$

Man beachte, daß die rechten Seiten dieser drei Ausdrücke auf der ganzen Komplexebene definiert sind, obwohl die linken Seiten nur auf der Halbebene $\{\operatorname{Re}(z)>0\}$ gelten. Mit anderen Worten, die Funktionen der rechten Seiten sind nichts andres als die analytischen Fortsetzungen der linken Integrale, deren Gebiet nur die rechte Halbebene ist.

Die obigen konfluent hypergeometrischen Funktionen sind auch mit den Hankelschen Funktionen einer komplexen Variablen darzustellen: z. B.

$$
\begin{aligned}
& G_{1}\left(\frac{1}{2}, 2 ; 2 i z\right)=-2 e^{i z\left\{H_{1}^{(2)}(z)+i H_{0}^{(2)}(z)\right\}} \\
& G_{1}\left(\frac{3}{2}, 3 ; 2 i z\right)=e^{i z H_{1}^{(2)}(z)}
\end{aligned}
$$

Nach den Integraldarstellungen (2) werden die Ableitungen der konfluent hypergeometrischen
Funktionen wieder mit denselben dargestellt:

$$
\begin{aligned}
G_{1}{ }^{\prime}\left(\frac{1}{2}, 2 ; z\right)= & \frac{1}{4} G_{1}\left(\frac{3}{2}, 3 ; z\right) \\
G_{1}{ }^{\prime}\left(\frac{3}{2}, 3 ; z\right)= & \left(1-\frac{2}{z}\right) G_{1}\left(\frac{3}{2}, 3 ; z\right) \\
& +\frac{2}{z} G_{1}\left(\frac{1}{2}, 2 ; z\right)
\end{aligned}
$$

Das Benehmen dieser Funktionen in der Nähe des unendlich fernen Punktes wird mit Hilfe der asymptotischen Formel kurz beschrieben; im besonderen

$$
\begin{aligned}
& G_{1}\left(\frac{1}{2}, 2 ; z\right)=\frac{4 i}{\sqrt{\pi}} z^{-1 / 2}+O\left(z^{-3 / 2}\right) \\
& G_{1}\left(\frac{3}{2}, 3 ; z\right)=-\frac{8 i}{\sqrt{\pi}} z^{-3 / 2}+O\left(z^{-5 / 2}\right) .
\end{aligned}
$$

\section{(2) Komplexe Theodorsensche Funktion}

Die Theodorsensche Funktion $C(k)$ wird folgenderweise definiert:

$$
C(k)=\frac{H_{1}^{(2)}(k)}{H_{1}^{(2)}(k)+i H_{0}^{(2)}(k)}
$$

wobei die Veränderliche $k$ reell ist. Lassen wir definieren

$$
\S(z)=-\frac{z}{2} \cdot \frac{G_{1}\left(\frac{3}{2}, 3 ; 2 z\right)}{G_{1}\left(\frac{1}{2}, 2 ; 2 z\right)}
$$

und nennen sie die verallgemeinerte Theodorsensche Funktion oder einfach die komplexe $\mathrm{T}$ Funktion, weil ihr Argument $z$ komplex ist.

Nach den Beziehungen (3) ist eine andere Definition möglich durch die Hankelschen Funktionen vom komplexen Argument:

$$
\S(z)=\frac{H_{1}^{(2)}(-i z)}{H_{1}^{(2)}(-i z)+i H_{0}^{(2)}(-i z)}
$$

Man kann obige zwei Definitionen nach den Umständen treffend gebrauchen. Der erstere Ausdruck scheint bequemer für die analytische Untersuchung. Noch dazu da die komplexe Zahl $z$ in dieser Arbeit eine physikalisch deutliche Bedeutung besitzt, so ist der erstere theoretisch günstiger. Aber wegen der historischen Umstände, daß die Hankelschen Funktionen weit genauer untersucht worden sind als die konfluent hypergeometrischen Funktionen, ist der letztere Ausdruck nützlich zu gebrauchen, um numerische Werte von der komplexen T-Funktion zu berechnen.

Außerdem hat man die Beziehung zwischen der Theodorsenschen Funktion und der komplexen T-Funktion. Wenn das komplexe Argu- 
ment $z$ besonders rein imaginär ist, stimmt diese mit jener überein; d.i.

$$
\Subset(i k)=C(k) \cdots \cdots k \in \boldsymbol{R}
$$

Offenbar ist die komplexe T-Funktion eine analytische Funktion, was folgenderweise anders gesagt werden kann: Die komplexe T-Funktion $\mathfrak{S}(z)$ ist gerade die analytische Fortsetzung der Theodorsenschen Funktion $C(k)$.

Die Theodorsensche Funktion ist, von mathematischem Standpunkt aus, eine lahme Funktion, deren Argument begrenzt reell ist, obwohl sie selbt komplexe Werte besitzt, währenddessen ist die komplexe T-Funktion eine vollkommene analytische Funktion.

Jetzt lassen wir über den analytischen Charakter der komplexen T-Funktion zusammenfassen. Nach (5) und (7) ist $\Subset(z)=$ $\frac{1}{2}+O\left(z^{-1}\right)$ wenn $z$ unendlich groß wird.

Nach den Integraldarsetllungen (2) sind $G_{1}\left(\frac{1}{2}, 2 ; \bar{z}\right)=-\overline{G_{1}\left(\frac{1}{2}, 2 ; z\right)}$ und $G_{1}\left(\frac{3}{2}, 3 ; \bar{z}\right)$ $=-\overline{G_{1}\left(\frac{3}{2}, 3 ; z\right)}$, wenn $\operatorname{Re}(z)$ positiv ist, was nach der Eigenschaft der analytischen Fortsetzung gilt überall, wo die Funktionen analytisch sind. Deshalb muß auf der ganzen Ebene

$$
\mathbb{S}(\bar{z})=\overline{\mathbb{S}(z)}
$$

Besonders wenn $z$ reell ist, ist auch $\Subset(z)$ reell.

Nach (4) und (7) wird die Ableitung von $\&(z)$ wieder mit derselben dargestellt:

$$
\begin{aligned}
& \mathfrak{E}^{\prime}(z)=\frac{1}{z}\{\S(z)\}^{2}+\left(2-\frac{1}{z}\right) \Subset(z)-1 \\
& \mathbb{E}^{\prime \prime}(z)=\frac{2}{z^{2}}\left[\{\Subset(z)\}^{3}-2\left\{(\mathbb{C}(z)\}^{2}+\mathbb{\complement}(z)\right]\right. \\
& +\frac{1}{z}\left[6\{\Subset(z)\}^{2}-6 \Subset(z)+1\right]+4 \Subset(z)-2
\end{aligned}
$$

Wenn einmal numerische Werte der Besselschen Funktionen $J_{0}(z)$ und $J_{1}(z)$ und der Neumannschen Funktionen $Y_{0}(z)$ und $Y_{1}(z)$ für komplexe Veränderliche $z$ bekannt sind, so ist

$$
\Subset(i z)=\frac{J_{1}(z)-i Y_{1}(z)}{\left\{J_{1}(z)-i Y_{1}(z)\right\}+i\left\{J_{0}(z)-i Y_{0}(z)\right\}}
$$

Numerische Werte dieser Besselschen Funktionen werden gewöhnlich gegen $\rho$ und $\phi$ gegeben (2), wobei $z=\rho e^{i \phi}$.

Schreibt man $\mathbb{E}(i z)=\mathbb{}\left(\rho e^{i(\phi+(\pi / 2))}\right)=\mathbb{E}(\rho: \phi)$,

$$
\begin{aligned}
\mathbb{E}(\rho: \phi+\varepsilon)= & \mathbb{E}(\rho: \phi)-\rho e^{i \phi \mathbb{S}^{\prime}}(\rho: \phi) \varepsilon \\
& +\frac{1}{2} \rho^{2} e^{2 i \phi \mathbb{E}^{\prime \prime}}(\rho: \phi) \varepsilon^{2}+\cdots
\end{aligned}
$$

Nach (11) sind

$$
\begin{aligned}
& \rho e^{i \phi \mathbb{\complement}^{\prime}(\rho: \phi)=i\left\{\Subset(\rho: \phi)-\mathbb{\mho}^{2}(\rho: \phi)\right\}} \\
& +\rho e^{i \phi}\{2 \Subset(\rho: \phi)-1\} \\
& \rho^{2} e^{2 i \phi \mathbb{C}^{\prime \prime}(\rho: \phi)} \\
& =-2\left\{\aleph^{3}(\rho: \phi)-2 \mathbb{\mho}^{2}(\rho: \phi)+\mathbb{E}(\rho: \phi)\right\} \\
& -i \rho e^{i \phi}\left\{6 \mathfrak{S}^{2}(\rho: \phi)-6 \mathfrak{E}(\rho: \phi)+1\right\} \\
& +2 \rho^{2} e^{2 i \phi}\{2 \Subset(\rho: \phi)-1\}
\end{aligned}
$$

Mittelst dieser Ausdrücke und vorhandener numerischer Tafel der Zylinderfunktionen komplexer Veränderlichen kann der Wert der komplexen T-Funktion gegen viele verschiedene Werte des Arguments genug genau ausgerechnet werden.

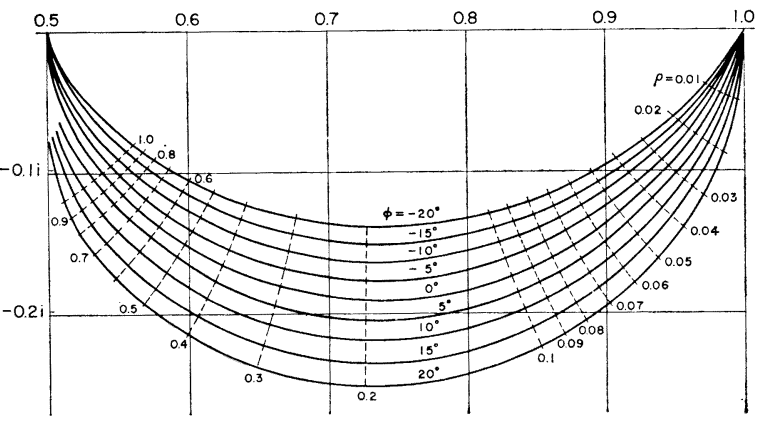

Bild 1 Verallgemeinerte Theodorsensche Funktion.

\section{(3) Integraltransformationen}

Wenn eine Funktion $u$ eines reellen Arguments einige mathematisch erforderliche Bedingungen erfüllt, werden ihre Fouriersch Transformierte $\mathfrak{F} u$, Fouriersch Umkehrstransformierte $\mathfrak{F}^{*} u$ und Laplacesch Transformierte $\_u$ definiert, wobei die ersteren zwei ein reelles Argument und währenddessen die Laplacesch Transformierte ein komplexes Argument haben.

$$
\begin{aligned}
& (\mathfrak{F} u)(k)=\frac{1}{\sqrt{2 \pi}} \int_{-\infty}^{\infty} u(t) e^{-i k t} d t \\
& \left(\mho^{*} u\right)(k)=\frac{1}{\sqrt{2 \pi}} \int_{-\infty}^{\infty} u(t) e^{i k t} d t \\
& (\Omega u)(p)=\int_{0}^{\infty} u(t) e^{-p t} d t
\end{aligned}
$$

Nach der Fourierschen Umkehrsformel $\mathfrak{F}^{*}(\mathfrak{F} u)=$ $\mathfrak{F}\left(\mathfrak{F}^{*} u\right)=u$, und nach der Laplaceschen Umkehrsformel $u(t)=\frac{1}{2 \pi i} \int_{\sigma-i \infty}^{\sigma+i \infty} e^{t p}(\Omega u)(p) d p$, wobei reelle Zahl $\sigma$ gewöhnlich beliebig bestimmt werden kann.

Diese Operation verwandeln lineare Differentialgleichungen mit konstanten Koeffizienten in lineare algebraische Gleichungen, weil 


$$
\left[\mathfrak{F}\left(\frac{d u}{d t}\right)\right](k)=i k(\mathfrak{F} u)(k), \quad \text { u. s. w. }
$$

Noch dazu wenn $u$ mit einer Faltungsform dargestellt ist, so gilt ein Theorem, das in diesem Studium eine große Rolle spielt.

$$
\begin{gathered}
\text { Wenn } u(x)=\int_{0}^{\infty} f(x-y) g(y) d y \text { ist, so sind } \\
(\mathfrak{F u})(k)=(\mathfrak{F} f)(k) \int_{0}^{\infty} g(y) e^{-i k y} d y \\
\left(\mathfrak{F}^{*} u\right)(k)=\left(\mathfrak{F}^{*} f\right)(k) \int_{0}^{\infty} g(y) e^{i k y} d y
\end{gathered}
$$

und wenn $u(x)=\int_{0}^{x} f(x-y) g(y) d y$ ist, so ist $(\Omega u)(p)$ $=(\Omega f)(p) \cdot(\Omega g)(p)$.

Die Funktion $u$ muß auch einige mathematische Bedingungen erfüllen, damit obige Formeln gelten. Zum Beispiel wenn $u(t)$ eine dauernd schwingende Quantität ist, die sich nicht zusammenzieht, so existiert weder $\int_{-\infty}^{\infty}|u(t)| d t$ noch $\int_{-\infty}^{\infty}|u(t)|^{2} d t$ mehr. Darum existiert (₹̛u) oder $\left(\mathfrak{F}^{*} u\right)$ im allgemeinen nicht, trotzdem darf man auch obige Formeln gebrauchen, wenn man sie für eine Distribution hält. Wenn die dauernd schwingende Funktion $u(t)$ eine wirkliche physikalische Quantität darstellt, so könnte man noch einige andere mathematische Bedingungen von $u$ voraussetzen, z. B. (1) Stetigkeit von $u(t)$ und ihrer Ableitungen nötiger Glieder, (2) Beschränktheit von $u$. Für diesen Fall kann man dafür halten, daß $u$ zum Funktionenraum $\mathcal{S}^{\prime}$ von "müßigen Distributionen" gehört, und man kann $\mathfrak{F} u$ und $\mathfrak{F}^{*} u$ definieren und deshalb obige Formeln gebrauchen.

\section{KLASSISCHE FLATTERNSCHWIN- GUNG}

\section{(1) Theodorsensche Theorie}

Lassen wir zuerst die Theorie klassischer Flatternschwingung kurz nachprüfen, um den Punkt unseres Studiums einzuführen.

Es ist wohl bekannt, daß kein Widerstand auf beliebigen in reibungsfreier Flüssigkeit gesetzten Körper wirkt. Wenn es aber Zirkulation $I$ um den Körper gibt, so wirkt ein Auftrieb $L=$ $\rho U \Gamma$, was als Theorie von Kutta und Joukowski bekannt ist. Nun denken wir weiter darüber nach, wie sich diese Formel verändert, wenn der Körper nicht mehr stillsteht sondern mit einer kleinen Amplitude schwingt. Diese Fragestellung ist zweifellos bedeutungslos, wenn die
Schwingung eine große Amplitude hat, wobei der Strömungsstand der Hinterseite des Körpers die Hypothese idealer Flüssigkeit gar nicht mehr erlaubt. Wir können die Form $L(t)=\rho U \Gamma(t)$ nicht hoffen, trotzdem könnten wir die Modifikation linearerweise darstellen, weil wir die von der Schwingung erregte Strömungsstörung für winzig klein ansehen; nämlich

$$
L(t)=\rho U\{\Gamma(t)+\gamma(t)\}
$$

Es gelang $\mathrm{T}$. Theodorsen, $\gamma(t) \mathrm{zu}$ bestimmen, für den Fall, daß ebene Platte harmonisch schwingt. Wenn die Platte schwingt, so schwingt natürlich auch die Größe der Zirkulation. Aber nach dem Erhaltungsgesetz des Wirbelbetrags fließt ihre Differenz als Wirbelstraße geradlinig hinterwärts weg, und .

$$
\dot{\Gamma}(t)+U \cdot \gamma_{w}(b, t)=0
$$

Nach der Theorie von Potentialströmung wird die normale Komponente $w_{a}$ der Strömungsgeschwindigkeit an der Tragfläche mit Hilfe von den Dichten $\gamma_{a}$ tragender Wirbel und $\gamma_{w}$ freier Wirbel ausgedrückt:

$$
\begin{aligned}
w_{a}(x, t)= & -\frac{1}{2 \pi} \oint_{-b}^{b} \frac{\gamma_{a}(\xi, t)}{x-\xi} d \xi \\
& -\frac{1}{2 \pi} \int_{b}^{\infty} \frac{\gamma_{w}(\xi, t)}{x-\xi} d \xi
\end{aligned}
$$

wobei $2 b$ gleich Sehne ist.

Läßt man den Wirbelbetrag auf der Wirbelstraße sich nicht vermindern, so gilt eine Art Homogenität

$$
\gamma_{w}(x, t)=\gamma_{w}\left(\xi, t+b \frac{\xi-x}{U}\right) .
$$

Das ist nicht anders als Lösung der Gleichung von Idealität der Flüssigkeit:

$$
U \frac{\partial \gamma_{w}}{\partial x}+\frac{\partial \gamma_{w}}{\partial t}=0
$$

Nun lassen wir uns beschränken auf den Fall, daß alle Quantitäten, die an unserem Phänomen teilnehmen, mit einer gleichen Kreisfrequenz $\omega$ rein periodisch schwingen: D.i.

(I ) vertikale Verschiebung $b h(t)=b h_{0} e^{i \omega t}$ rotationale Verschiebung $\varphi(t)=\varphi_{0} e^{i \omega t}$

$$
\begin{aligned}
& \gamma_{a}(\xi, t)=\gamma_{a}(\xi) e^{i \omega t} \\
& \gamma_{w}(\xi, t)=\gamma_{w}(\xi) e^{i \omega t} \\
& w_{a}(x, t)=w_{a}(x) e^{i \omega t}
\end{aligned}
$$

(III) Zirkulation

$$
\Gamma(t)=\Gamma_{0} e^{i \omega t}
$$

Auftrieb

$$
L(t)=L_{0} e^{i \omega t}
$$

Drehmoment $M(t)=M_{0} e^{i \omega t}$

wobei nur die reellen Teile dieser Ausdrücke Bedeutung haben, was $z u$ rechtfertigen ist, weil 
folgende Berechnungen alle linear sind.

Die Bedeutung dieser Voraussetzung wird nachher genau überlegt. Die Leser sollten nur bemerken, daß hier nur die zwei Bedingungen erster Gruppe wesentlich und die übrigen nicht mehr als von sekundärer Bedeutung sind.

Nach (13), (14) und (15)

$$
\begin{aligned}
& \frac{1}{2 \pi} \oint_{-1}^{1} \frac{\gamma_{a}(\xi)}{x-\xi} d \xi \\
& \quad=\frac{i \omega \Gamma_{0}}{2 \pi U} e^{i k} \int_{1}^{\infty} \frac{e^{-i k \xi}}{x-\xi} d \xi-w_{a}(x)
\end{aligned}
$$

wobei $x$ statt $\frac{x}{b}$ und $\xi$ statt $\frac{\xi}{b}$ geschrieben worden sind, d.h. die Raumveränderlichen sind hiernach ohne Dimension. Die dimensionlose Größe $k=\frac{\omega b}{U}$ wird "reduzierte Frequenz" genannt.

Nach dem Theorem von Söhngen gilt

$$
\begin{array}{r}
f(x)=-\frac{1}{\pi^{2}} \oint_{-1}^{1} \sqrt{\frac{1-\xi}{1+\xi}} \frac{d \xi}{x-\xi} \\
\times \oint_{-1}^{1} \sqrt{\frac{1+\eta}{1-\eta}} \frac{f(\eta)}{\xi-\eta} d \eta,
\end{array}
$$

wenn $f(1)<\infty$ ist.

Wendet man diese Umkehrsformel auf (16) an,

$$
\begin{array}{r}
\gamma_{a}(x)=\frac{2}{\pi} \sqrt{\frac{1-x}{1+x}}\left\{\oint_{-1}^{1} \sqrt{\frac{1+\xi}{1-\xi}} \frac{w_{a}(\xi)}{x-\xi} d \xi\right. \\
\left.+\frac{i \omega \Gamma_{0}}{2 U} e^{i k} \int_{1}^{\infty} \sqrt{\frac{\eta+1}{\eta-1}} \frac{e^{-i k \eta}}{x-\eta} d \eta\right\} . \cdots
\end{array}
$$

Die Druckdifferenz $\Delta p_{a}(x, t)$ zwischen unten und oben wird nach der Theorie von Potentialströmung mit Hilfe von $\gamma_{a}$ berechnet:

$$
\begin{aligned}
& \frac{\Delta p_{a}(x, t)}{\rho U}=-\gamma_{a}(x, t)-\frac{b}{U} \int_{1}^{x} \frac{\partial \gamma_{a}}{\partial t}(\xi, t) d \xi \\
& \frac{\Delta p_{a}(x)}{\rho U}=-\gamma_{a}(x)-i k \int_{1}^{x} \gamma_{a}(\xi) d \xi, \quad \ldots \ldots . .
\end{aligned}
$$

wobei $\Delta p_{a}(x, t)=\Delta p_{a}(x) e^{i \omega t}$.

$$
\begin{aligned}
\therefore \quad \frac{L_{0}}{\rho U b}= & -2 C(k) \int_{-1}^{1} \sqrt{\frac{1+\xi}{1-\xi}} w_{a}(\xi) d \xi \\
& -2 i k \int_{-1}^{1} \sqrt{1-\xi^{2}} w_{a}(\xi) d \xi \quad \ldots . \\
\frac{M_{0}}{\rho U b^{2}}= & \{1-C(k)\} \int_{-1}^{1} \sqrt{\frac{1+\xi}{1-\xi}} w_{a}(\xi) d \xi \\
& -2 \int_{-1}^{1} \sqrt{1-\xi^{2}} w_{a}(\xi) d \xi \\
& +i k \int_{-1}^{1} \xi \sqrt{1-\xi^{2}} w_{a}(\xi) d \xi
\end{aligned}
$$

Vorausgesetzt, daß keine Trennung erzeugt wird an der Platte, muß die normale Komponente $w_{a}$ mit der vertikalen Geschwindigkeit der Platte übereinstimmen, und also

$$
\begin{aligned}
w_{a}(x, t)= & -\{b h(t)+U \varphi(t)+x \dot{\varphi}(t)\} \\
\therefore \quad \frac{L_{0}}{\pi \rho U^{2} b}= & \left\{2 i k C(k)-k^{2}\right\} h_{0} \\
& +\{(2+i k) C(k)+i k\} \varphi_{0} \\
\frac{M_{0}}{\pi \rho U^{2} b^{2}}= & i k C(k) h_{0} \\
& +\left\{\left(1+\frac{i}{2} k\right) C(k)+\frac{1}{8} k^{2}-\frac{i k}{2}\right\} \varphi_{0}
\end{aligned}
$$

Wirken eine elastische Widerstandskraft $b K_{h} h(t)$ gegen die Verschiebung $b h(t)$ und ein elastisches Widerstandsmoment $K_{\phi} \varphi(t)$ gegen die Drehverschiebung $\varphi(t)$, so sind

$$
\begin{gathered}
-L(t)=m b \ddot{h}(t)+b K_{h} h(t) \\
M(t)=I \ddot{\varphi}(t)+K_{\phi} \varphi(t),
\end{gathered}
$$

wobei $m$ Masse der Platte und $I$ Trägheitsmoment der Platte in bezug auf die Achse $\{x=0\}$ sind.

Noch ein Faktor fehlt. Das ist Strukturdämpfung. Um sie in Betracht $z u$ ziehen, braucht man nur $K_{h}$ durch $K_{h}\left(1+i g_{h}\right)$ und $K_{\phi}$ durch $K_{\phi}\left(1+i g_{\phi}\right) \mathrm{zu}$ ersetzen in den Bewegungsgleichungen $^{3)}$. So sind

$$
\left.\begin{array}{c}
-L(t)=m b\left\{\ddot{h}(t)+\omega_{h}^{2}\left(1+i g_{h}\right) h(t)\right\} \\
M(t)=I\left\{\ddot{\varphi}(t)+\omega_{\phi}{ }^{2}\left(1+i g_{\phi}\right) \varphi(t)\right\},
\end{array}\right\}
$$

wobei $\omega_{h}=\sqrt{\frac{K_{h}}{m}}$ und $\omega_{\phi}=\sqrt{\frac{K_{\phi}}{I}}$ eigene Kreisfrequenz der Vertikalschwingung bzw. die der Drehschwingung sind. Substituiert man (15) für diese Gleichungen, werden die Bewegungsgleichungen schließlilh lineare und homegene, algebraische Gleichungen für $h_{0}$ und $\varphi_{0}$.

Damit die Platte wirklich schwingt, muß die Koeffizientendeterminante gleich Null sein.

$\mathrm{Da}$ sie eine Gleichung auf dem Körper der komplexen Zahlen ist, entscheidet sie zwei reelle Zahlen, nämlich die reduzierte Frequenz $k$ und die kritische Windgeschwindigkeit $U$. So kann man die kritische Geschwindigkeit der nicht gestörten Luftströmung im Sinn entscheiden, daß die Platte bei ihr rein periodisch schwingt. Diese kritische Windgeschwindigkeit wird gewöhnlich "Flattern (wind) geschwindigkeit" genannt und oft mit $U_{F}$ bezeichnet.

\section{(2) Theorie klassischer Flatternschwingungen}

Lassen wir hier kurz das Skelett der Theorie klassischer Flatternschwingungen und auch ihre Fragen anordnen:

(1) Die Platte schwingt mit so kleiner Amplitude, daß die Theorie von Potentialströmung und 
der $K-J$ Satz darauf anzuwenden sind.

(2) Wegen der Helmholtzschen Sätze über Wirbelbewegungen, fährt die zeitliche Differenz der Zirkulation als ein Wirbelschicht an, und jedes von ihren Elementen, seinen Wirbelbetrag zeitlich konstant haltend, fließt mit der Strömungsgeschwindigkeit hinterwärts weg.

(3) Man begrenzt seinen Gegenstand nur auf den Fall, daß alle Quantitäten, die sich bei der Schwingung der Platte verändern, mit einer gleichen Frequenz harmonisch schwingen. Das ist mit (15) analytisch dargestellt worden.

(4) Amplituden des Auftriebs und des Kraftmoments werden bestimmt. Substituiert man dieses Ergebnis für die Bewegungsgleichungen erreicht man ein lineares Gleichungssystem in $h_{0}$ und $\varphi_{0}$.

(5) Existenzfähigkeit von nicht trivialen $h_{0}$ und $\varphi_{0}$ verlangt einige spezielle Werte der Strömungsgeschwindigkeit. Ihr Minimum, das gewöhnlich Flatternwindgeschwindigkeit oder kritische Windgeschwindigkeit genannt wird, ist eine der wichtigsten Charakteristiken. Weil diese Existenzfähigkeit auch von Strukturdämpfung des Systems abhängig ist, ist ihre Bedingung z.B. gegen Windgeschwindigkeit und Strukturdämpfungskonstante zu bezeichnen, gewöhnlich wie das als Beispiel angeführte Bild.

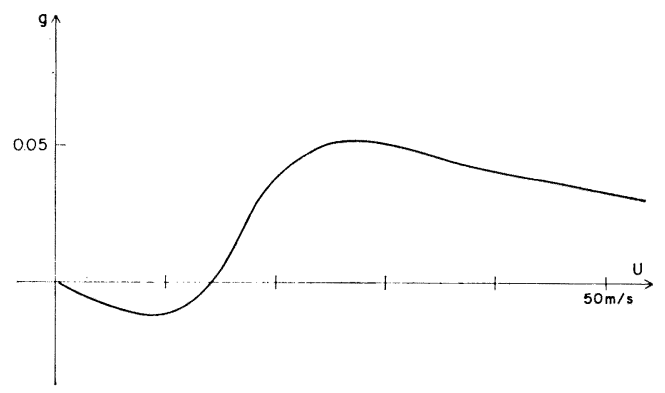

Bild 2 Ergebnis der Theodorsenschen Methode.

Man könnte aber folgenderweise sagen: Die Theodorsensche Theorie bleibt noch eine halbempirische Formel, die die mit (15) dargestellte empirische Tatsache als ihren Grund ohne Kritik aufgenommen hat. Es ist nicht erklärt worden, warum oder auf welchen Fall denn diese Phänomena sich verwirklichen. Lassen wir nun das Objekt unserer Betrachtungen folgenderweise zusammenfassen.

(I) Prüfung der Existenz von Schwingungsformen anderer Art als die mit (15) dargestellte rein periodische Schwingung: Es ist nicht nur theoretisch sondern auch praktisch unentbehrlich, festzustellen, daß keine Schwingungen solcher Form existieren können, damit man die Flatterngeschwindigkeit mit kristischer Windgeschwindigkeit identifizieren darf. Nach unseren experimentellen Ergebnissen, stimmen die Frequenzen beider Schwingungen, vertikale und rotationale, nicht überein, wenigstens wenn die Luftströmung aus irgendeinem Grund gestört ist. Natürlich können wir darauf rechnen, daß sie gewiß übereinstimmen, wenn die Platte sich in ungestörter Strömung befindet, was aber theoretisch festzustellen ist.

(II) Die auf dem Bild 2 aufgezeichnete Kurve stellt die Bedingung dar, unter der die mit (15) dargestellte Schwingung existieren kann. Aber in welchem Bewegungszustand ist denn die Platte, wenn sie in anderen Umständen liegt ?

Im Fall von Experimenten in Windkanal und vielleicht auch im Fall von natürlichem Wind, wird die Flatterngeschwindigkeit nicht plötzlich sondern allmählich realisiert. Und zwar es läßt sich kaum erwarten, daß die Windgeschwindigkeit bei unserem Phänomen, ganz anders als bei dem der Flugmechanik, auf lange Zeit immer einen konstanten Wert hält. Bewegungszustand an den Punkten außer der aufgezeichneten Kurve hat also für uns besondere Bedeutung im Vergleich mit für Flugmechaniker, denn unsere Objekte, verschiedene Baustrukturen, müssen unter Bewegung des Naturwindes stehen bleiben. Diese zweite Aufgabe verlangt uns gewiß eine mathematische Erweiterung klassischer Flatterntheorie. Die Flatterntheorie bestimmt die Existenzbedingung einer besonderen aber zugleich der wichtigsten Schwingung. Unsre neue Theorie muß aber die Schwingungsform gegen beliebige Umgebungslage bestimmen können. Lassen wir sie nachher einfach Stabilitätstheorie heißen.

Wenn zum Beispiel die Windgeschwindigkeit $U$ und die Strukturdämpfungskonstante $g$ veränderlich sind, so muß die Stabilitätstheorie irgendeine Funktionalbeziehung der Art $f(U, g$; Schwingungsform $)=0$ bringen. Und wenn dieser Ausdruck gegen die besonderen $U$ und $g$, die nach der Flatterntheorie bestimmt worden ist, die die rein periodische Schwingung (15) als der Schwingungsform bestimmt, könnten wir ja die Stabilitäts theorie für eine Erweiterung der Flatterntheorie halten.

Unser Ziel setzt sich also aus nächsten zwei Angaben zusammen:

(A) Bejahende Antwort auf die erste Frage, nämlich Feststellung der Einzigartigkeit der Lösung (15) unter den Hypothesen (1) und (2).

(B) Antwort auf die zweite Frage, nämlich Bildung der Stabilitätstheorie. 
Es ist bemerkenswürdig, daß diese zwei Angaben ausschließlich mathematisch gelöst werden können, und auch daß die zweite Antwort während des Studiums erster Angabe auf ganz natürliche Weise eingeführt wird.

Die erste und zweite Angaben sind eine Art physikalische Voraussetzungen, und wir nehmen sie so wie sie sind auf, wie wir uns in der Vorbemerkung darauf beschränkt haben.

Anderseits, der Prozeß von der tritten Angaben an ist Analyse unseres physikalischen Modells, deren Gültigkeit wir prüfen möchten. Aber, genauer gesagt, die tritte Angabe fällt über alles in unsere Augen, denn die übrigen folgen ihr auf natürlicher Art. Wir müßten deshalb ein bißchen genau betrachten, welchen Charakter denn diese Angabe trägt.

Da gebraucht man eine typische mathematische Methode der Art, daß man jede von den Quantitäten, die eigentlich eine Art Funktion sind, durch eine komplexe Zahl ersetzt, um die fundamentalen Gleichungen, die eigentlich Funktionengleichungen sind und doch sich in der Flatterntheorie nicht offen zeigen, in algebraische Gleichungen $z u$ verwandeln. Und zwar diese Annahme hat die Phänomene, die wirklich beobachtet werden, richtig ausgedrückt.

\section{ANALYTISCHES VERFAHREN DER FLATTERNTHEORIE}

\section{(1) Analytische Darstellung der Theodorsen- schen Theorie}

Es ist schon erwähnt worden, daß fundamentale Gleichungen mit Hilfe der Annahme (15) von Theodorsen sich verbergend gelöst worden sind. Aber wir lassen sie hier auftreten. Diese fundamentalen Gleichungen, d.i. analytische Darstellung der Theodorsenschen Theorie, spielt eine große Rolle nicht nur bei der Prüfung erster Angabe und beim Bilden der Stabilitätstheorie sondern auch, bei der Analysis der Phänomene unter Wirkung des irgendetwas gestörten Windes.

Weil wir die ersten zwei Angaben der Theodorsenschen Methode aufgenommen haben, können wir sofort die Anfangsausdrücke (12), (13) und (14) empfangen:

$$
\begin{gathered}
\frac{d \Gamma}{d t}\left(t-b \frac{\xi-1}{U}\right)+U \cdot \gamma_{w}(\xi, t)=0 \\
w_{a}(x, t)=-\frac{1}{2 \pi} \oint_{-1}^{1} \frac{\gamma_{a}(\xi, t)}{x-\xi} d \xi \\
-\frac{1}{2 \pi} \int_{1}^{\infty} \frac{\gamma_{w}(\xi, t)}{x-\xi} d \xi
\end{gathered}
$$

wobei die zwei Raumveränderlichen $x$ und $\xi$ dimensionlos sind.

Nach der Söhngenschen Umkehrsformel

$$
\begin{gathered}
\gamma_{a}(\xi, t)=\frac{2}{\pi} \sqrt{\frac{1-\xi}{1+\xi}} \oint_{-1}^{1} \sqrt{\frac{1+x}{1-x}} \frac{w_{a}(x, t)}{\xi-x} d x \\
-\frac{1}{\pi U} \sqrt{\frac{1-\xi}{1+\xi}} \int_{1}^{\infty} \sqrt{\frac{\eta+1}{\eta-1}} \frac{\dot{\Gamma}\left(t-b \frac{\eta-1}{U}\right)}{\eta-\xi} d \eta \\
\therefore \quad \Gamma(t)=-2 b \int_{-1}^{1} \sqrt{\frac{1+x}{1-x}} w_{a}(x, t) d x \\
-\frac{b}{U} \int_{1}^{\infty}\left(\sqrt{\frac{\eta+1}{\eta-1}}-1\right) \dot{\Gamma}\left(t-b \frac{\eta-1}{U}\right) d \eta
\end{gathered}
$$

Nach (19)

$$
\begin{aligned}
\frac{L(t)}{\rho U}=\Gamma(t)+\frac{b}{U} \dot{\Gamma}(t)-\frac{b^{2}}{U} \int_{-1}^{1} \xi \dot{\gamma}_{a}(\xi, t) d \xi \\
\frac{M(t)}{\rho U b}=-\frac{b}{2 U} \dot{\Gamma}(t)-b \int_{-1}^{1} \xi \gamma_{a}(\xi, t) d \xi \\
+\frac{b^{2}}{2 U} \int_{-1}^{1} \xi^{2} \dot{\gamma}_{a}(\xi, t) d \xi
\end{aligned}
$$

Substituiert man (27) für diese zwei,

$$
\begin{aligned}
\frac{L(t)}{\rho U}= & \Gamma(t)+\frac{b}{U} \dot{\Gamma}(t) \\
& -\frac{b^{2}}{U^{2}} \int_{1}^{\infty}\left(\eta-\sqrt{\eta^{2}-1}\right) \ddot{\Gamma}\left(t-b \frac{\eta-1}{U}\right) d \eta \\
& -2 \frac{b^{2}}{U} \int_{-1}^{1} \sqrt{1-x^{2}} \dot{w}_{a}(x, t) d x \\
\frac{M(t)}{\rho U b}= & -\frac{b}{2 U} \dot{\Gamma}(t) \\
& -\frac{b}{U} \int_{1}^{\infty}\left(\eta-\sqrt{\eta^{2}-1}\right) \dot{\Gamma}\left(t-b \frac{\eta-1}{U}\right) d \eta \\
& -\frac{b^{2}}{2 U^{2}} \int_{1}^{\infty}\left(\eta \sqrt{\eta^{2}-1}-\eta^{2}+\frac{1}{2} \sqrt{\frac{\eta+1}{\eta-1}}\right) \\
& \times \ddot{\Gamma}\left(t-b \frac{\eta-1}{U}\right) d \eta \\
& -\frac{b^{2}}{2 U} \int_{-1}^{1} \sqrt{\frac{1+x}{1-x}} \dot{w}_{a}(x, t) d x \\
& -2 b \int_{-1}^{1} \sqrt{1-x^{2}} w_{a}(x, t) d x \\
& +\frac{b^{2}}{U} \int_{-1}^{1} x \sqrt{1-x^{2}} \dot{w}_{a}(x, t) d x
\end{aligned}
$$

Nach (23)

$$
\begin{aligned}
\Gamma(t) & +\frac{b}{U} \int_{1}^{\infty}\left(\sqrt{\frac{\eta+1}{\eta-1}}-1\right) \dot{\Gamma}\left(t-b \frac{\eta-1}{U}\right) d \eta \\
& =2 \pi b\{b \dot{h}(t)+U \varphi(t)\}+\pi b^{2} \dot{\varphi}(t) \quad \cdots \cdots(30)
\end{aligned}
$$




$$
\begin{aligned}
\frac{L(t)}{\rho U}= & \Gamma(t)+\frac{b}{U} \dot{\Gamma}(t) \\
& -\frac{b^{2}}{U^{2}} \int_{1}^{\infty}\left(\eta-\sqrt{\eta^{2}-1}\right) \ddot{\Gamma}\left(t-b \frac{\eta-1}{U}\right) d \eta \\
& +\frac{\pi b^{2}}{U}\{b \ddot{h}(t)+U \dot{\varphi}(t)\} \\
\frac{M(t)}{\rho U b}= & -\frac{b}{2 U} \dot{\Gamma}(t) \\
& -\frac{b}{U} \int_{1}^{\infty}\left(\eta-\sqrt{\eta^{2}-1}\right) \dot{\Gamma}\left(t-b \frac{\eta-1}{U}\right) d \eta \\
& -\frac{b^{2}}{2 U^{2}} \int_{1}^{\infty}\left(\eta \sqrt{\eta^{2}-1}-\eta^{2}+\frac{1}{2} \sqrt{\frac{\eta+1}{\eta-1}}\right) \\
& \times \ddot{\Gamma}\left(t-b \frac{\eta-1}{U}\right) d \eta \\
& +\pi b\{b \dot{h}(t)+U \varphi(t)\} \\
& +\frac{\pi b^{2}}{2 U}\left\{b \ddot{h}(t)+U \dot{\varphi}(t)+\frac{b}{4} \ddot{\varphi}(t)\right\} \quad \cdots(32)
\end{aligned}
$$

Lassen wir hier zwei spezielle Lösungen dieser Gleichungen kurz ansehen:

(1) Wenn die Platte stillsteht, verschwinden alle Ableitungen der fundamentalen Gleichungen, und $\Gamma=2 \pi b U \varphi, \quad L=\rho U \Gamma=2 \pi \rho b U^{2} \varphi, \quad M=\pi \rho b^{2} U^{2} \varphi$, was gewiß Resultat der statischen Theorie ist.

(2) Wegen der speziellen Form der fundamentalen Gleichungen kann man auf spezielle Lösungen rechnen, bei denen alle unbekannten Funktionen gleichzeitig eine Form von $e^{z t}$ ( $z=$ komplex) zeigen. Wenn $\operatorname{Re}(z)$ positiv ist, konvergieren sicher alle Integrale der Gleichungen. Diese konvergieren auch wenn $\operatorname{Re}(z)=0$ ist, weil ihre Kerne alle $O\left(\frac{1}{\eta}\right)$ sind, wenn $\eta$ unendlich groß wird. Man könnte leicht voraussehen, daß diese Lösung der Theorie von Theodorsen entspricht. In der Tat, wenn man (15) für die fundamentalen Gleichungen substituieren,

$$
\begin{aligned}
& \Gamma_{0}=2 i b U \frac{e^{-i k}}{k} \frac{2 i k h_{0}+(2+i k) \varphi_{0}}{H_{1}^{(2)}(k)+i H_{0}^{(2)}(k)} \\
& \frac{L_{0}}{\rho U}=-\frac{\pi i}{2} k e^{i k} H_{1}^{(2)}(k) \Gamma_{0}+\pi b U\left(-k^{2} h_{0}+i k \varphi_{0}\right) \\
& \frac{M_{0}}{\rho U b}=-\pi k e^{i k\left[\frac{1}{4} H_{0}^{(2)}(k)\right.} \\
& \left.+\frac{k}{8}\left\{H_{1}^{(2)}(k)+i H_{0}^{(2)}(k)\right\}\right] I_{0}^{\prime} \\
& \quad+\pi b U\left\{\left(i k-\frac{k^{2}}{2}\right) h_{0}+\left(1+\frac{i k}{2}-\frac{k^{2}}{8}\right) \varphi_{0}\right\}
\end{aligned}
$$

Diese Ausdrücke haben wesentlich nur eine spezielle Funktion; nämlich sie können mit einigen elementaren Funktionen und einer Funktion, die nur vom Verhältnis der zwei Hankelschen Funktionen abhängig ist, dargestellt werden. Im besonderen kann man die Theodorsensche Funktion als diese parametrische Funktion wählen, und

$$
\begin{gathered}
L_{0}=2 \pi \rho U^{2} b C(k)\left\{i k h_{0}+\left(1+\frac{i k}{2}\right) \varphi_{0}\right\} \\
+\pi \rho U^{2} b\left\{-k^{2} h_{0}+i k \varphi_{0}\right\} \\
M_{0}=\pi \rho U^{2} b^{2} C(k)\left\{i k h_{0}+\left(1+\frac{i k}{2}\right) \varphi_{0}\right\} \\
-\pi \rho U^{2} b^{2}\left\{\frac{i k}{2}-\frac{k^{2}}{8}\right\} \varphi_{0}
\end{gathered}
$$

Diese sind nicht anders als das Ergebnis von Theodorsen. Also kann man folgenderweise erklären: "Die Theodorsensche Theorie entspricht einer von möglichen Lösungen der fundamentalen Gleichungen ".

Auf diese Weise haben wir nun die Frage (I) natürlicherweise ergriffen. Nämlich wir brauchen nur zu prüfen, ob das Ergebnis der Theodorsenschen Theorie denn einzig mögliche Lösung der fundamentalen Gleichungen ist.

$\mathrm{Zu}$ diesem $\mathrm{Zweck}$ hat man die fundamentalen Gleichungen mit dimensionloser Form auszudrücken.

Lassen wir $\frac{U t}{b}$ aufs neue mit $t$ bezeichnen.

Dieses neue $t$ ist dimensionlose Zeitveränderliche. Bezeichnet die Ableitung betreffs des neuen $t$ mit Gedankenstrich, $\quad f^{\prime}(t)=\frac{b}{U} \dot{f}$. Bezeichnen wir weiter dimensionlose Größen $\frac{\Gamma(t)}{2 \pi U b}, \frac{M(t)}{2 \pi \rho U^{2} b}$ und $\frac{M(t)}{2 \pi \rho U^{2} b^{2}}$ von neuem $\Gamma(t), L(t)$ und $M(t)$,

$$
\Gamma(t)+\int_{0}^{\infty} f_{1}(s) \Gamma^{\prime}(t-s) d s=h^{\prime}(t)+\varphi(t)+\frac{1}{2} \varphi^{\prime}(t)
$$

$$
\begin{aligned}
L(t)= & \Gamma(t)+\Gamma^{\prime}(t)-\int_{0}^{\infty} f_{2}(s) \Gamma^{\prime \prime}(t-s) d s \\
& +\frac{1}{2} h^{\prime \prime}(t)+\frac{1}{2} \varphi^{\prime}(t) \\
M(t)= & -\frac{1}{2} \Gamma^{\prime}(t)-\int_{0}^{\infty} f_{2}(s) \Gamma^{\prime}(t-s) d s \\
& -\frac{1}{2} \int_{0}^{\infty} f_{3}(s) \Gamma^{\prime \prime}(t-s) d s \\
& +\left\{\frac{1}{4} h^{\prime \prime}(t)+\frac{1}{2} h^{\prime}(t)\right\} \\
& +\left\{\frac{1}{16} \varphi^{\prime \prime}(t)+\frac{1}{4} \varphi^{\prime}(t)+\frac{1}{2} \varphi(t)\right\}
\end{aligned}
$$

wobei

$$
f_{1}(x)=\sqrt{\frac{x+2}{x}}-1
$$




$$
\begin{aligned}
f_{2}(x)= & -\sqrt{(x+1)^{2}-1}+(x+1) \\
f_{3}(x)= & (x+1) \sqrt{(x+1)^{2}-1}-(x+1)^{2} \\
& +\frac{1}{2} \sqrt{\frac{x+2}{x}}
\end{aligned}
$$

Wir haben schon geäußert, daß sie fein sind, nicht nur aus äußerlichem Grund, sondern auch in analytischem Sinn. Denn analytische Eigenschaften unserer Theorie haben in der dimensionlosen Form deutlich erscheint, was wir nachher sehen. Aus diesem Grund lassen wir diese dimensionlose Form einfach fundamentale Gleichungen heißen. Es ist $z u$ beachten, daß ihre Kerne alle die Art haben, daß $f_{r}(x)=O\left(\frac{1}{x}\right)(r=1,2,3)$ bei $x \rightarrow \infty$.

Die fundamentalen Gleichungen sind lineare Integrodifferentialgleichungen, deren Integrale alle bestimmte Integrale über $(0, \infty)$ sind und eine Art Faltungsform besitzen, was ihre analytisch entscheidendste Eigenschaft ist. Leicht kann man sehen, daß diese schöne Eigenschaft auf die Bedingung (14) oder am Ende auf unsere Voraussetzung von "reibungsfreien Flüssigkeit" hinauskommt.

Wenn die kinematische Zähigkeit $\nu$ der Flüssigkeit nicht $\mathrm{zu}$ vernachlässigen ist, so muß man statt (14) aufnehmen

$$
\begin{aligned}
\gamma_{w}(\xi, t)=- & \frac{1}{4 \sqrt{\pi \nu}} \int_{0}^{t} \exp \left[-\frac{\{b(\xi-1)-U s\}^{2}}{4 \nu s}\right] \\
& \times\left(1+\frac{b \xi}{U s}\right) \dot{\Gamma}(t-s) d s,
\end{aligned}
$$

was mit unserer Bedingung übereinstimmt nur wenn $\nu$ verschwindet, weil

$$
\lim _{\nu \rightarrow 0} \frac{\exp \left\{-\frac{(x-U t)^{2}}{4 \nu t}\right\}}{\sqrt{\nu t}}=2 \frac{\sqrt{\pi}}{U} \delta(x-U t) .
$$

\section{(2) Bewegungsgleichungen}

Die Bewegungsgleichungen (24) können folgenderweise dimensionlos ausgedrückt werden:

$$
\left.\begin{array}{c}
-L(t)=\frac{\mu}{2}\left\{h^{\prime \prime}(t)+k_{h^{2}}\left(1+i g_{h}\right) h(t)\right\} \\
M(t)=\frac{\mu r^{2}}{2}\left\{\varphi^{\prime \prime}(t)+{k_{\phi}}^{2}\left(1+i g_{\phi}\right) \varphi(t)\right\}
\end{array}\right\}
$$

wobei $\quad \mu=\frac{m}{\pi \rho b^{2}}, \quad r^{2}=\frac{I}{m b^{2}}, \quad k_{h}=\frac{b \omega_{h}}{U} \quad$ und

$$
k_{\phi}=\frac{b \omega_{\phi}}{U} .
$$

Aber man muß beachten, daß sie auf beliebige Schwingungen nicht direkt angewandt werden dürfen, weil sie eigentlich nur bei rein periodischen Schwingungen gelten, deren Frequenz wesentlich positiv ist. Um sie $z u$ erweitern, hat man die von allgemeiner Form schwingende Funktion $u(t)$ in harmonisch schwingende Elemente zu zerlegen.

$$
u(t)=\int_{-\infty}^{\infty} \frac{\hat{u}(\omega)}{\sqrt{2 \pi}} e^{i \omega t} d \omega
$$

Die Größe der Strukturdämpfungskraft, die bei der rein periodischen $\hat{u}(\omega) e^{i \omega t}$ wirkt, ist gleich $i g_{u} K_{u} \hat{u}(\omega) e^{i \omega t}$, wenn die Kreisfrequenz $\omega$ positiv ist. Wenn diese dagegen negativ ist, so muß die Kraft gleich $-i g_{u} K_{u} \hat{u}(\omega) e^{i \omega t}$ sein. Die gesamte Dämpfungskraft ist also gleich

$$
\begin{aligned}
& i g_{u} K_{u} \int_{0}^{\infty} \frac{\hat{u}(\omega)}{\sqrt{2 \pi}} e^{i \omega t} d \omega \\
& \quad-i g_{u} K_{u} \int_{-\infty}^{0} \frac{\hat{u}(\omega)}{\sqrt{2 \pi}} e^{i \omega t} d \omega \\
& \quad \equiv g_{u} K_{u} \int_{-\infty}^{\infty} i *(\omega) \frac{\hat{u}(\omega)}{\sqrt{2 \pi}} e^{i \omega t} d \omega
\end{aligned}
$$

Diese Tatsache ist folgenderweise $z u$ formulieren:

$$
\left.\begin{array}{rl}
-L(t) & =\frac{\mu}{2}\left\{h^{\prime \prime}(t)+k_{h^{2}}\left(1+i^{*} g_{h}\right) h(t)\right\} \\
M(t) & =\frac{\mu r^{2}}{2}\left\{\varphi^{\prime \prime}(t)+k_{\phi^{2}}\left(1+i^{*} g_{\phi}\right) \varphi(t)\right\},
\end{array}\right\}
$$

wobei

$$
\left.\begin{array}{l}
\mathfrak{F}(i * u)(k)=i \operatorname{sign}(k)(\mathfrak{F} u)(k) \\
\mathfrak{F}^{*}\left(i^{*} u\right)(k)=i \operatorname{sign}(k)\left(\mathfrak{F}^{*} u\right)(k) \\
\mathfrak{L}\left(i^{*} u\right)(p)=i \operatorname{sign}\left\{I_{m}(p)\right\}(\mathfrak{R} u)(p)
\end{array}\right\}
$$

Es ist klar, daß die Dämpfungskraft gleich Null ist, wenn die Frequenz der Schwingung gleich Null ist, und auch daß jene stetigerweise klein wird, wenn diese sich Null nähert, was entspricht, daß der Bewegungsproze $\beta$ reversibel sein muß. Die Formeln (39) müssen also in der Nähe der Null-Frequenz ein bißchen verändert werden. Diese vielmehr abstrakte Modifikation macht aber nichts in folgenden Diskussionen, weil wir nachher nur arithmetische Berechnungen und Integralrechnung gebrauchen und nie differentieren; trotzdem ist sie nötig, um unser Argument mathematisch genau auszuführen.

\section{(3) Einzigkeit der Lösungen der fundamentalen Gleichungen}

Die fundamentalen Gleichungen und die Bewegungsgleichungen sind nun vorhanden, und wir können anfangen, sie $z u$ lösen. Ihre jede unbekannte Funktion kann, wie schon erwähnt, für 
eine der Distributionen $\mathcal{S}^{\prime}$ gehalten werden, und wir können ihre Fouriersch Transformierten annehmen. Schreibt man diese fett und eliminiert man die transformierte Zirkulation,

$$
\left.\begin{array}{rl}
\boldsymbol{L}(k)= & \left\{i k C(k)-\frac{1}{2} k^{2}\right\} \boldsymbol{h}(k) \\
& +\left\{\left(1+\frac{1}{2} i k\right) C(k)+\frac{1}{2} i k\right\} \boldsymbol{\phi}(k) \\
\boldsymbol{M}(k)= & \frac{1}{2} i k C(k) \boldsymbol{h}(k) \\
& +\left\{\frac{1}{2}\left(1+\frac{1}{2} i k\right) C(k)-\frac{1}{4} i k+\frac{1}{16} k^{2}\right\} \boldsymbol{\phi}(k)
\end{array}\right\}
$$

Die Zeitveränderliche in den fundamentalen Gleichungen ist gleich $\frac{U}{b} t$, wobei $t$ die wirkliche Zeitveränderliche ist. Die neue Veränderliche $k$ ist also gleich $\frac{b \omega}{U} ;$ d.i. die reduzierte Frequenz ist natürlicherweise eingeführt worden.

Die Bewegungsgleichungen werden

$$
\left.\begin{array}{c}
-\boldsymbol{L}(k)=\frac{\mu}{2}\left\{k_{h^{2}}\left(1+i^{*} g_{h}\right)-k^{2}\right\} \boldsymbol{h}(k) \\
\boldsymbol{M}(k)=\frac{\mu r^{2}}{2}\left\{k_{\phi}^{2}\left(1+i^{*} g_{\phi}\right)-k^{2}\right\} \boldsymbol{\phi}(k)
\end{array}\right\}
$$

und unsere Gleichungen haben am Ende folgende Form.

$$
\left[\begin{array}{cc}
2 i k C(k)-k^{2} & (2+i k) C(k)+i k \\
+\mu\left\{k_{h^{2}}\left(1+i^{*} g_{h}\right)-k^{2}\right\} & \left(1+\frac{1}{2} i k\right) C(k)-\frac{1}{2} i k+\frac{1}{8} k^{2} \\
i k C(k) & -\mu r^{2}\left\{k_{\phi}^{2}\left(1+i^{*} g_{\phi}\right)-k^{2}\right\}
\end{array}\right]
$$

Die Koeffizientendeterminante $A(k)$ dieser Gleichung ist analytisch abgesehen von dem originalen Punkt und dem unendlich fernen Punkt. Da $A(k)$ einen hinkenden Charakter hat, daß sie trotz ihres reellen Arguments komplexe Werte besitzt, bleibt hier ihre mathematische Eigenschaft noch unklar. Aber wir verstehen später, daß $A(k)$ nichts andres als die Beschränkte einer analytischen Funktion $\mathfrak{A}(z)$ einer komplexen Variablen auf die imaginäre Achse ist. Wenn analytischer Charakter von $\mathfrak{A}(z)$ erklärt wird, werden der von $A(k)$ und also mathematische Bedeutung der Theodorsenschen Methode erschlossen.

Lassen wir untersuchen, wie es sich mit den Nullstellen von $A(k)$ verhält.
(1) Wenn $A(k)$ keine Nullstelle besitzt;

Da (42) homogen ist, verschwinden $\boldsymbol{h}(k)$ und $\phi(k)$ identisch als Funktionen von $k$. Also sind $h(t)$ und $\varphi(t)$ gleich Null als Distributionen. Aber weil $h(t)$ und $\varphi(t)$, in Rücksicht auf ihre physikalische Bedeutung, stetige Quantitäten sind, so müssen sie identisch gleich Null als gewöhnliche Funktionen sein.

(2) Wenn $A(k)$ Nullstellen besitzt:

Da $C(k)=O(1)$ für große Werte von $k$ ist, ist es klar, daß $k$ von genug großen Absolutbeträgen nicht Nullstelle von $A(k)$ sein können. Also befinden die Nullstellen sich in einem beschränkten Gebiet mit Ausnahme vom originalen Punkt. Und zwar diese Nullstellen sind voneinander isoliert, weil $A(k)$ in diesem Gebiet analytisch ist.

In genug kleinem Intervall jeder Nullstelle $c$ wird $A(k)$ um $c$ Taylorsch entwickelt und man kann schreiben $A(k)=(k-c) A(c ; k)$, wobei $A(c ; k)$ eine reguläre Funktion von $k$ in diesem Intervall ist. Jedes Element der Unterdeterminantenmatrix $\Delta(k)$ ist reguläre Funktion von $k$ in diesem Interval.

Multipliziert man die beiden Seiten von (42) mit $\Delta(k)$,

$$
A(c ; k)\left[\begin{array}{c}
(k-c) h(k) \\
(k-c) \phi(k)
\end{array}\right]=\left[\begin{array}{l}
0 \\
0
\end{array}\right] .
$$

Wenn die Nullstelle $c$ einfach ist, verschwindet $A(c ; k)$ nicht in diesem Intervall, und also

$$
\left.\begin{array}{l}
(k-c) \boldsymbol{h}(k)=0 \\
(k-c) \phi(k)=0 .
\end{array}\right\}
$$

Die beiden Distributionen $\boldsymbol{h}(k)$ und $\boldsymbol{\phi}(k)$ sind also eine Multiplikation von $\delta(k-c)$ mit beliebiger Konstanten. Wendet man die Fouriersche Umkehrstransformation an, findet man leicht, daß $h(t)$ und $\varphi(t)$ von der Form $\left\{\right.$ Konstante $\left.\cdot e^{i c t}\right\}$ sind. Aber da $h(t)$ und $\varphi(t)$ selbstverständlich stetige Quantitäten sind, haben sie die Form

$$
\begin{aligned}
& h(t)=\mathrm{Konstante} \cdot e^{i c t} \\
& \varphi(t)=\mathrm{Konstante} \cdot e^{i c t}
\end{aligned}
$$

als gewöhnliche Funktionen.

Nach der Funktionentheorie sind die Nullstellen voneinander isoliert. Und wie schon erwähnt, kann weder der unendlich ferne Punkt noch der originale Punkt Nullstelle sein. Bei unserem Fall gibt es also höchstens nur endliche Nullstellen, und $h(t)$ und $\varphi(t)$ sind lineare Kombinationen der den Nullstellen entsprechenden rein periodischen Schwingungen.

Es ist leider noch nicht festgestellt worden, ob $A(k)$ nur einfache Nullstellen hat. Auf den pathologischen Fall, daß die $A(k)$ konstituierenden 
Quantitäten miteinander in spizieller Beziehung stehen, könnte $A(k)$ mehrfache Nullstellen haben. In diesem Fall gelten statt (43)

$$
\left.\begin{array}{l}
(k-c)^{n} \boldsymbol{h}(k)=0 \\
(k-c)^{n} \boldsymbol{\phi}(k)=0,
\end{array}\right\}
$$

und es ist auf diesen Fall $\mathrm{zu}$ beweisen, daß $\boldsymbol{h}(k)$ und $\phi(k)$ lineare Kombinationen von $\delta(k-c)$, $\delta^{\prime}(k-c), \ldots$ und $\delta^{(n-1)}(k-c)$ sind. Zum Schluß sind also $h(t)$ und $\varphi(t)$ lineare Kombinationen von $e^{i c t}, t e^{i c t}, \cdots, t^{n-2} e^{i c t}$ und $t^{n-1} e^{i c t}$.

Darum muß die Form der Lösung (15) etwas modifiziert werden, wenn die Gleichung $A(k)=0$ vielfache Wurzeln besitzt. Aber selbst diese sozusagen pathologischen Lösungen werden nach der Gleichung $A(k)=0$ bestimmt. In diesem Sinn kann man erklären, daß die Theodorsensche Lösung die einzig mögliche Lösung der fundamentalen Gleichungen ist, was bedeutet, daß unsere erste Aufgabe (I) bejahend gelöst worden ist.

\section{STABILITATSTHEORIE}

\section{(1) Fundamentale Gleichungen}

Lassen wir hier beginnen, die Stabilitätstheorie zusammenzusetzen. Die Theodorsensche Theorie bringt die Existenzbedingung einer Schwingung von spezieller Form, rein periodische Schwingung. Aber, logisch gesagt, sie bestimmt nie die Stabilitätsbedingung der Bewegung. Die Theodorsensche Feststellung der kritischen Windgeschwindigkeit ist wie folgt auszudrücken:

$U=U_{F} \Longleftrightarrow$ Eine rein periodische Schwingung existiert.

Die Bedingung von Periodizität ist aber hier gleichgültig und nur die von Dauer der Schwingung, d.i. sie weder dämpft noch divergiert, ist wesentlich wichtig, weil jene unvermeidlich aus dieser folgt, wie wir schon gesehen haben.

Die sozusagen kritische Windgeschwindigkeit ist also nichts mehr als die Windgeschwindigkeit, die die rein periodische Schwingung erlaubt; trotzdem heißen wir sie die kritische Windgeschwindigkeit, denn wir vermuten empirisch, daß die Platte gefährlich an der Geschwindigkeit von über diesen Wert sei. Dieses ist aber gerade nichts andres als die allgemeine Logik, die Stabilität von Schwingungen $z \mathrm{u}$ bestimmen. Nämlich man hat folgenderweise $\mathrm{zu}$ formulieren:

(1) $U$ ist in sicherem Gebiet, wenn jede Schwingung, die von beliebiger kleiner Störung erregt worden ist, dämpft immer bei der Windgeschwindigkeit $U$.

(2) $U$ ist in gefährlichem Gebiet, wenn irgend mindestens eine kleine Störung kann die Schwingung bei der Windgeschwindigkeit $U$ allmählich divergieren lassen.

(3) Der Grenzwert der beiden Gebiete wird die kritische Windgeschwindigkeit genannt.

Lassen wir diese Idee genauer feststellen, um Anhalt für die Stabilitätstheorie zu gewinnen.

Zur Erreichung dieses $Z$ wecks geben wir der Platte kleine Störung an irgendeinem beliebigen Zeitpunkt, und beobachten seine folgend entstehende Bewegung. Wählt man diesen Zeitpunkt zu $t$ gleich Null, so schwingt die Platte, die während negatives $t$ stillstehend war, seit $t$ gleich Null. Der Fall, daß die Platte an $t$ gleich Null schon in Bewegung ist, wird nachher geprüft, und man sieht dabei, daß es keinen Einfluß auf unseren Schluß ausübt, wie der Anfangszeitpunkt gewählt wird.

Auf dieselbe Weise wie der letzte Abschnitt erreicht man ein neues System der fundamentalen Gleichungen:

$$
\begin{aligned}
\Gamma(t)+ & \frac{b}{U} \int_{1}^{1+(U t / b)}\left(\sqrt{\frac{\eta+1}{\eta-1}}-1\right) \dot{\Gamma}\left(t-b \frac{\eta-1}{U}\right) d \eta \\
=2 \pi b & \{b \dot{h}(t)+U \varphi(t)\}+\pi b^{2} \dot{\varphi}(t) \\
\frac{L(t)}{\rho U}= & \Gamma(t)+\frac{b}{U} \dot{\Gamma}(t) \\
& -\frac{b^{2}}{U^{2}} \int_{1}^{1+(U t / b)}\left(\eta-\sqrt{\eta^{2}-1}\right) \ddot{\Gamma}\left(t-b \frac{\eta-1}{U}\right) d \eta \\
& +\frac{\pi b^{2}}{U}\{b \ddot{h}(t)+U \dot{\varphi}(t)\} \\
\frac{M(t)}{\rho U b}= & -\frac{b}{2 U} \dot{\Gamma}(t) \quad \cdots \cdots(46) \\
& -\frac{b}{U} \int_{1}^{1+(U t / b)}\left(\eta-\sqrt{\eta^{2}-1}\right) \dot{\Gamma}\left(t-b \frac{\eta-1}{U}\right) d \eta \\
& -\frac{b^{2}}{2 U^{2}} \int_{1}^{1+(U t / b)}\left(\eta \sqrt{\eta^{2}-1}-\eta^{2}+\frac{1}{2} \sqrt{\frac{\eta+1}{\eta-1}}\right) \\
& \times \ddot{\Gamma}\left(t-b \frac{\eta-1}{U}\right) d \eta+\pi b\{b \dot{h}(t)+U \varphi(t)\} \\
& +\frac{\pi}{2} \frac{b^{2}}{U}\left\{b \ddot{h}(t)+U \dot{\varphi}(t)+\frac{b}{4} \ddot{\varphi}(t)\right\} \quad \cdots \cdots(47)
\end{aligned}
$$

Stellt man sie mit dimensionloser Form dar,

$$
\begin{aligned}
\Gamma(t)+\int_{0}^{t} f_{1}(s) \Gamma^{\prime}(t-s) d s=h^{\prime}(t)+\varphi(t)+\frac{1}{2} \varphi^{\prime}(t) \\
L(t)=\Gamma(t)+\Gamma^{\prime}(t)-\int_{0}^{t} f_{2}(s) \Gamma^{\prime \prime}(t-s) d s \\
+\frac{1}{2} h^{\prime \prime}(t)+\frac{1}{2} \varphi^{\prime}(t) \\
M(t)=-\frac{1}{2} \Gamma^{\prime}(t)-\int_{0}^{t} f_{2}(s) \Gamma^{\prime}(t-s) d s
\end{aligned}
$$




$$
\begin{aligned}
& -\frac{1}{2} \int_{0}^{t} f_{3}(s) \Gamma^{\prime \prime}(t-s) d s+\left\{\frac{1}{4} h^{\prime \prime}(t)+\frac{1}{2} h^{\prime}(t)\right\} \\
& +\left\{\frac{1}{16} \varphi^{\prime \prime}(t)+\frac{1}{4} \varphi^{\prime}(t)+\frac{1}{2} \varphi(t)\right\}
\end{aligned}
$$

Nun ist die Beziehung zwischen der Theorie klassischer Flatternschwingungen und der Stabilitätstheorie klar gemacht worden; nämlich nur die Integralgrenzen der beiden Systeme sind voneinander verschieden, was folgenderweise $\mathrm{zu}$ erklären ist: Der Übergang von der Flatterntheorie zur Stabilitätstheorie entspricht dem vom bestimmten Integral zum unbestimmten Integral.

Wenn die Schwingung schon begonnen hat, als die kleine Störung gegeben wird, so gibt es schon eine Wirtelreihe, Anfangswirbelreihe, hinter dem schwingenden Körper, und die abgeänderte Form der fundamentalen Gleichungen ist

$$
\begin{aligned}
& \Gamma(t)+\int_{0}^{t} f_{1}(s) \Gamma^{\prime}(t-s) d s=\cdots+C_{1}(t) \\
& L(t)=\cdots+C_{2}(t) \\
& M(t)=\cdots+C_{3}(t)
\end{aligned}
$$

Die punktierten Linien zeigen die Glieder der ursprünglichen Gleichungen (48), (49) und (50), und

$$
\begin{aligned}
C_{1}(t)= & \frac{1}{2 \pi U} \int_{t}^{\infty} f_{1}(s) \gamma_{i}(t-s) d s \\
C_{2}(t)= & \frac{1}{2 \pi U} \int_{t}^{\infty} f_{2}(s) \gamma_{i}{ }^{\prime}(t-s) d s \\
C_{3}(t)= & \frac{1}{2 \pi U} \int_{t}^{\infty} f_{2}(s) \gamma_{i}(t-s) d s \\
& +\frac{1}{4 \pi U} \int_{t}^{\infty} f_{3}(s) \gamma_{i}{ }^{\prime}(t-s) d s,
\end{aligned}
$$

wobei $\gamma_{i}$ Dichte der Anfangswirbelreihe ist. Sie sind von den unbekannten $h(t), \varphi(t)$ und $\Gamma(t)$ unabhängige, festgesetzte Funktionen von $t$.

\section{(2) Lösungen der Stabilitätstherie}

Die unbekannten Funktionen der fundamentalen Gleichungen können mit Hilfe der Laplaceschen Transformation abgetrennt werden. Schreibt man ihre Laplacesch Transformierten fett, eliminiert man die transformierte Zirkulation und drückt man die Laplace-transformierten von $f_{r}(r=1,2$, 3) mit den konfluent hypergeometrischen Funktionen aus,

$$
\begin{aligned}
& \boldsymbol{L}(p)=\left\{p \Subset(p)+\frac{1}{2} p^{2}\right\} \boldsymbol{h}(p) \\
& +\left\{\left(1+\frac{1}{2} p\right) \Subset(p)+\frac{1}{2} p\right\} \phi(p)+D_{L}(p) \\
& \boldsymbol{M}(p)=\frac{1}{2} p \Subset(p) \boldsymbol{h}(p)
\end{aligned}
$$

$$
\begin{aligned}
& +\left\{\frac{1}{2}\left(1+\frac{1}{2} p\right) \Subset(p)-\frac{1}{4} p-\frac{1}{16} p^{2}\right\} \phi(p)+D_{M}(p) \\
& D_{L}(p)=\frac{1}{p}\{1-\Subset(p)\} \Gamma_{0} \\
& +\left\{\frac{1}{p}+\frac{1}{p^{2}}+\frac{\pi i}{4} G_{1}\left(\frac{3}{2}, 3 ; 2 p\right)\right\} \Gamma_{1} \\
& \quad-\left\{\Subset(p)+\frac{p}{2}\right\} h_{0}-\frac{1}{2}\left[h_{1}+\{\Subset(p)+1\} \varphi_{0}\right] \\
& D_{M}(p)=\left[\frac{1}{2 p}\{1-\Subset(p)\}-\frac{\pi i}{16}\left\{p G_{1}\left(\frac{3}{2}, 3 ; 2 p\right)\right.\right. \\
& \left.\left.+(p+2) G_{1}\left(\frac{1}{2}, 2 ; 2 p\right)\right\}\right] \Gamma_{0}-\frac{1}{2} \Subset(p) h_{0}-\frac{1}{4} h_{1} \\
& \quad-\left[\frac { \pi i } { 8 } \left\{\left(1-\frac{2}{p}\right) G_{1}\left(\frac{3}{2}, 3 ; 2 p\right)\right.\right. \\
& \left.+\left(1+\frac{2}{p}\right) G_{1}\left(\frac{1}{2}, 2 ; 2 p\right)\right\} \\
& \left.\quad-\left(\frac{1}{2 p}+\frac{1}{p^{2}}+\frac{1}{p^{3}}\right)\right] \Gamma_{1}-\frac{1}{4}\left\{\Subset(p)-\frac{1}{4}\right\} \varphi_{0}-\frac{1}{16} \varphi_{1}
\end{aligned}
$$

Die Zeichen $h_{0}, h_{1}, \varphi_{0}$ u.s.w. drücken Anfangswerte aus, z.B. $h_{0}=h(0), h_{1}=h^{\prime}(0), \varphi_{0}=\varphi(0)$, u.s.w.

Die komplexe Veränderliche $p$ mußte positiven reellen Teil haben, damit die drei Integraltransformierten convergierten. (52) gilt aber nicht nur auf dieser Halbebene sondern auch auf der ganzen Komplexebene, was man folgenderweise erklären kann: Die Darstellung (52) ist die analytische Fortsetzung der Ausdrücke der Stabilitätstheorie.

Nach den Bewegungsgleichungen sind

$$
\begin{aligned}
-\boldsymbol{L}(p)= & \frac{\mu}{2}\left\{p^{2}+k_{h^{2}}\left(1+i^{*} g_{h}\right)\right\} \boldsymbol{h}(p)-\frac{\mu}{2}\left(p h_{0}+h_{1}\right) \\
\boldsymbol{M}(p)= & \frac{\mu r^{2}}{2}\left\{p^{2}+k_{\phi}^{2}\left(1+i * g_{\phi}\right)\right\} \boldsymbol{\phi}(p) \\
& -\frac{\mu r^{2}}{2}\left(p \varphi_{0}+\varphi_{1}\right)
\end{aligned}
$$

Die fundamentalen Gleichungen haben schließlich folgende Form.

$$
\begin{aligned}
& {\left[\begin{array}{rr}
a_{11}(p) & a_{12}(p) \\
a_{21}(p) & a_{22}(p)
\end{array}\right]\left[\begin{array}{l}
\boldsymbol{h}(p) \\
\boldsymbol{\phi}(p)
\end{array}\right]=\left[\begin{array}{c}
D_{h}(p) \\
D_{\phi}(p)
\end{array}\right] \ldots . } \\
& a_{11}(z)= 2 z \S(z)+z^{2}+\mu\left\{z^{2}+k_{h^{2}}\left(1+i^{*} g_{h}\right)\right\} \\
& a_{12}(z)=(2+z) \Subset(z)+z \\
& a_{21}(z)= z \S(z) \\
& a_{22}(z)=\left(1+\frac{1}{2} z\right) \Subset(z)-\frac{1}{2} z-\frac{1}{8} z^{2} \\
&-\mu r^{2}\left\{z^{2}+k_{\phi}{ }^{2}\left(1+i^{*} g_{\phi}\right)\right\}
\end{aligned}
$$




$$
\begin{aligned}
& D_{h}(z)=-2 D_{L}(z)-\mu\left(z h_{0}+h_{1}\right) \\
& D_{\phi}(z)=-2 D_{M}(z)-\mu r^{2}\left(z \varphi_{0}+\varphi_{1}\right)
\end{aligned}
$$

$D_{h}$ und $D_{\phi}$ sind für eine Darstellung der Anfangsstörung $\mathrm{zu}$ halten.

Löst man (53) nach $\boldsymbol{h}(p)$ und $\boldsymbol{\phi}(p)$ auf,

$$
\begin{aligned}
& \boldsymbol{h}(p)=\frac{a_{22}(p) D_{h}(p)-a_{12}(p) D_{\phi}(p)}{\mathfrak{A}(p)} \\
& \boldsymbol{\phi}(p)=\frac{a_{11}(p) D_{\phi}(p)-a_{21}(p) D_{h}(p)}{\mathfrak{Y}(p)} \\
& \mathfrak{X}(z)=a_{11}(z) a_{22}(z)-a_{12}(z) a_{21}(z)
\end{aligned}
$$

Obwohl die zwei Laplacesch Transformierten $\boldsymbol{h}(p)$ und $\phi(p)$ eigentlich nur auf der rechten Hälfte der Komplexebene definiert werden, werden diese Ausdrücke über die ganze Ebene wohl definiert.

Nach der Laplaceschen Umkehrsformel sind

$$
\left.\begin{array}{l}
h(t)=\frac{1}{2 \pi i} \int_{C} \frac{a_{22}(p) D_{h}(p)-a_{12}(p) D_{\phi}(p)}{\mathfrak{A}(p)} e^{p t} d p \\
\varphi(t)=\frac{1}{2 \pi i} \int_{C} \frac{a_{11}(p) D_{\phi}(p)-a_{21}(p) D_{h}(p)}{\mathfrak{A}(p)} e^{p t} d p
\end{array}\right\}
$$

Der Integrationsweg $C$ ist eine gerade, mit der imaginären Achse parallele Linie, und alle Nullstellen von $\mathfrak{U}(p)$ befinden sich auf der linken Seite von $C$. Das ist möglich, weil die Nullstellen sich in einem beschränkten Gebiet befinden, weil $\mathfrak{Y}(p)=O\left(p^{4}\right)$ in der Nähe des unendlich fernen Punktes. (Wenn $\mathfrak{U}(p)$ an der reellen Achse nicht stetig wäre, könnte man nicht solch einen Weg beliebig wählen. $\mathfrak{A}(p)$ ist nicht analytisch an der reellen Achse wegen der Singularität der Dämpfungskraft, trotzdem haben wir schon ihre Stetigkeit bestätigt in betreff des Ausdrucks (39).)

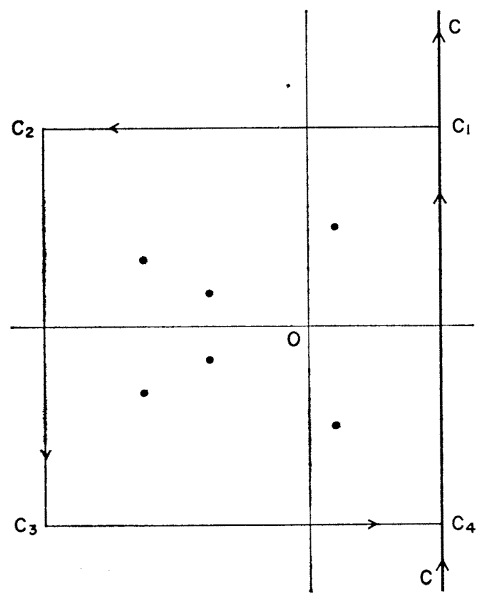

Bild 3 Integrationsweg.
Wegen der Eigenschaft der Integranden, in der Nähe des unendlich fernen Punktes genug schnell klein $z u$ werden, kann man eine genug große geschlossene Linie, die alle Nullstellen von $\mathfrak{X}(p)$ einschließt, als den Integrationsweg statt der geraden Linie aufnehmen. Nämlich der Zähler der Integranden ist in drittem Grad von $z$ und der Nenner ist in viertem Grad in der Nähe des unendlichen Punktes, und die Integrale über $C_{1} C_{2}$, $C_{2} C_{3}$ und $C_{3} C_{4}$ verschwinden, wenn $C_{1}, C_{2}, C_{3}$ und $C_{4}$ genug fern sind.

$$
\begin{aligned}
& \text { Also } \\
& \left.\begin{array}{l}
h(t)=\frac{1}{2 \pi i} \oint \frac{a_{22}(p) D_{h}(p)-a_{12}(p) D_{\phi}(p)}{\mathfrak{X}(p)} e^{p t} d p \\
\varphi(t)=\frac{1}{2 \pi i} \oint \frac{a_{11}(p) D_{\phi}(p)-a_{21}(p) D_{h}(p)}{\mathfrak{A}(p)} e^{p t} d p
\end{array}\right\}
\end{aligned}
$$

Lassen wir die Gleichung $\mathfrak{A}(z)=0$ wieder auch die Flatterngleichung nennen und ihre Wurzeln mit $p_{\nu}(\nu=0,1,2, \cdots, n)$ ausdrücken. Vorausgesetzt, daß die Nullstellen von $\mathfrak{X}(z)$ alle einfach sind, so sind nach dem allgemeinen Satz der Funktionentheorie

$$
\begin{aligned}
& h(t)=\sum_{\nu=1}^{n} \frac{a_{22}\left(p_{\nu}\right) D_{h}\left(p_{\nu}\right)-a_{12}\left(p_{\nu}\right) D_{\phi}\left(p_{\nu}\right)}{\mathfrak{U}^{\prime}\left(p_{\nu}\right)} e^{p_{\nu} t} \\
& \varphi(t)=\sum_{\nu=1}^{n} \frac{a_{11}\left(p_{\nu}\right) D_{\phi}\left(p_{\nu}\right)-a_{21}\left(p_{\nu}\right) D_{h}\left(p_{\nu}\right)}{\mathfrak{U}^{\prime}\left(p_{\nu}\right)} e^{p_{\nu} t} \\
& \mathfrak{U}^{\prime}\left(p_{\nu}\right)=\left[\frac{d \mathfrak{A}(z)}{d z}\right]_{z=p_{\nu}}
\end{aligned}
$$

Wenn jede Nullstelle negativen reellen Teil besitzt, so ist (60) eine sogenannte gedämpfte Schwingung und also stabil. Aber wenn es mindestens eine Nullstelle gibt, deren reeller Teil positiv ist, so steigt das ihr entsprechende Element $e^{p_{\nu} t}$ mit der Zeit exponential an, und also die Schwingung ist instabil. Diese Bestimmung von Stabilität der Schwingung wird nur von $\mathfrak{Y}(z)$, d.i. von der dynamischen Eigenschaft des Phänomens, besonders von der Windgeschwindigkeit $U$ herbeigeführt und ist von den Anfangsstörungen $D_{h}$ und $D_{\phi}$ unabhängig.

Nimmt man an, daß der Nullpunkt $p_{0}$ sich am rechtesten auf der Komplexebene befindet, nämlich daß sein reeller Teil maximal ist, so sind annährungsweise

$$
\left.\begin{array}{l}
h(t)=\frac{a_{22}\left(p_{0}\right) D_{h}\left(p_{0}\right)-a_{12}\left(p_{0}\right) D_{\phi}\left(p_{0}\right)}{\mathfrak{A}^{\prime}\left(p_{0}\right)} e^{p_{n} t} \\
\varphi(t)=\frac{a_{11}\left(p_{0}\right) D_{\phi}\left(p_{0}\right)-a_{21}\left(p_{0}\right) D_{h}\left(p_{0}\right)}{\mathfrak{U}^{\prime}\left(p_{0}\right)} e^{p_{0} t}
\end{array}\right\}
$$


Die Schwingung ist also stabil bzw. instabil, je nachdem der reelle Teil von $p_{0}$ negativ bzw. positiv ist, und die Schwingung ist also in kritischem Zustand, wenn $p_{0}$ sich auf der imaginären Achse befindet. In der Tat bewegt $p_{0}$ sich stetigerweise nach der Änderung der Windgeschwindigkeit und kreuzt die imaginäre Achse an speziellem Wert von $U$.

Diese Kennzeichnung der kritischen Schwingung entspricht dem Ergebnis der Flatterntheorie, wie wir erwarteten. Das ist folgenderweise leicht zu erklären: Auf diesen Fall ist $p_{0}$ gleich $i k$, das nach der Flatterngleichung $\mathfrak{A}(i k)=0$ entscheidet wird. Aber nach (42) und (53) kann man leicht sehen, daß die Unterdeterminantenmatrix von (42) gleich $\left[\begin{array}{ll}a_{11}(i k) & a_{12}(i k) \\ a_{21}(i k) & a_{22}(i k)\end{array}\right]$ ist und also $A(k)=\mathfrak{A}(i k)$. Nämlich die kritische Zahl $k$ der Stabilitätstheorie auch der Wurzel von $A(k)=0$ ist. Diese Beziehung zwischen den beiden Theorien ist folgenderweise $z u$ erklären: Die Stabilitätstheorie ist eine Erweiterung der Flatterntheorie; diese fängt nur eine spezielle, eine Art neutrale Lösung von jener.

Wenn $p_{0}$ eine mehrfache z.B. $m$-fache Nullstelle ist, so wird $e^{p_{0} t}$ von (61) nach dem Theorem von Residuum durch $t^{m-1} e^{p_{0} t}$ ersetzt. Obwohl es schwierig ist, die Existenzbedingung solcher mehrfacher Nullstellen theoretisch $\mathrm{zu}$ bestimmen, kann man numerisch leicht sehen, ob die Ableitungen von $\mathfrak{A}(z)$ gleich Null sind. Der mehrfache Wurzel steht also praktisch außer Frage.

Wenn es irgendeine Anfangswirbelreihe schon an $t=0$ gibt, gilt (51) statt (48), (49) und (50). Auf diesen Fall verändern sich nur $D_{L}(p)$ und $D_{M}(p)$ von (52), weil die Beifügungsglieder $C_{1}(t)$, $C_{2}(t)$ und $C_{3}(t)$ von den unbekannten Quantitäten unabhängig sind, und die Form der Flatterngleichung $\mathfrak{A}(z)$ bleibt also unveränderlich. Die Lage der Nullstellen steht nicht unter dem Einfluß von Existenz solcher Anfangswirbelreihe.

\section{(3) Numerisches Beispiel}

Wir nehmen hier ein folgendes Beispiel auf, um die Resultat dieses Studiums numerisch $z u$ prüfen:

$$
\begin{aligned}
& b=0.40 \mathrm{~m}, \quad \mu=133.5, \quad r^{2}=0.668, \\
& \omega_{h}=8.197 \mathrm{rad} / \mathrm{s}, \quad \omega_{\phi}=9.448 \mathrm{rad} / \mathrm{s}(\mathrm{A}) \\
& \text { oder } \omega_{\phi}=16.69 \mathrm{rad} / \mathrm{s}(\mathrm{B}) .
\end{aligned}
$$

Diese Größen entsprechen einem wirklichen Modell.

Da die Flatterngleichung die Form $f\left(U, g, p_{0}\right)$ $=0$ hat, bestimmt sie eine Beziehung unter vier reellen Größen, die Windgeschwindigkeit $U$, die Strukturdämpfungskonstante $g$, die Wachstumsrate $\sigma$ und die Kreisfrequenz $\omega$ der Schwingung, was folgenderweise symbolisch geschrieben werden kann;

$$
\begin{aligned}
& h(t)=h_{0} \cdot e^{\sigma(U, g) \cdot t} \cos \left\{\omega(U, g) \cdot t+\delta_{h}\right\} \\
& \varphi(t)=\varphi_{0} \cdot e^{\sigma(U, g) \cdot t} \cos \left\{\omega(U, g) \cdot t+\delta_{\phi}\right\}
\end{aligned}
$$

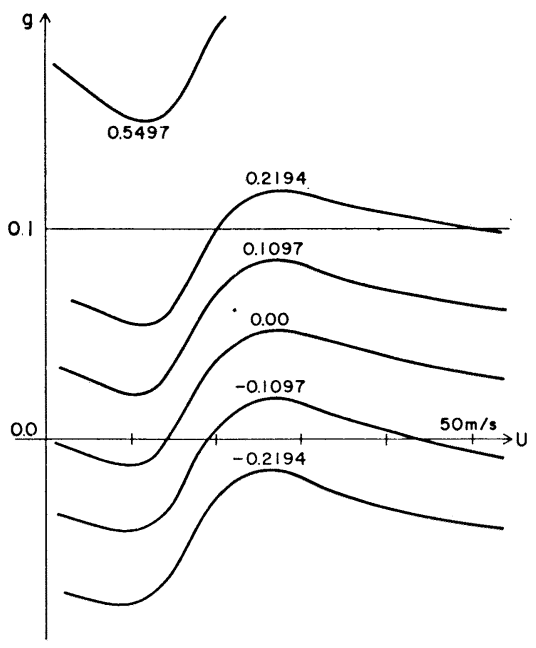

Bild 4-A $(U, g)$-Darstellung der Stabilitätstheorie.

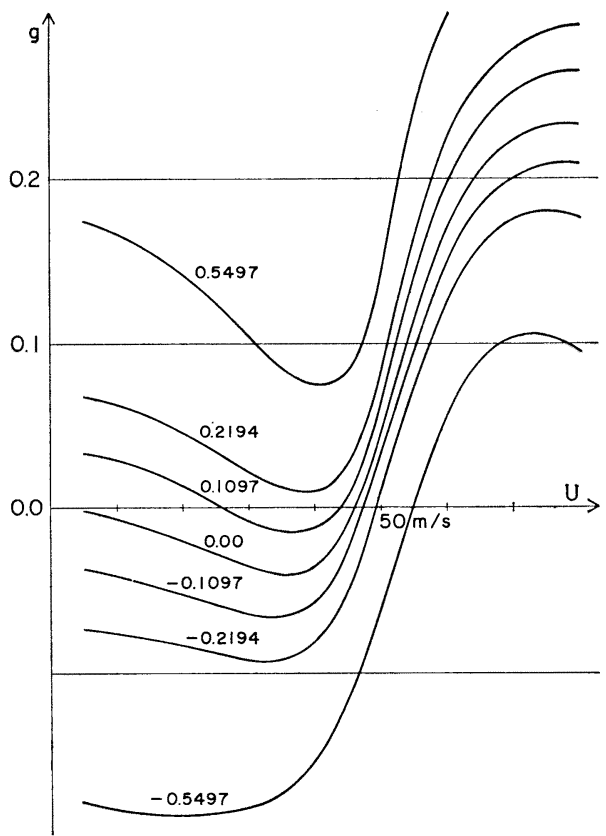

Bild 4-B $(U, g)$-Darstellung der Stabilitätstheorie. 
Es gäbe also viel Möglichkeit, die Beziehung darzustellen.

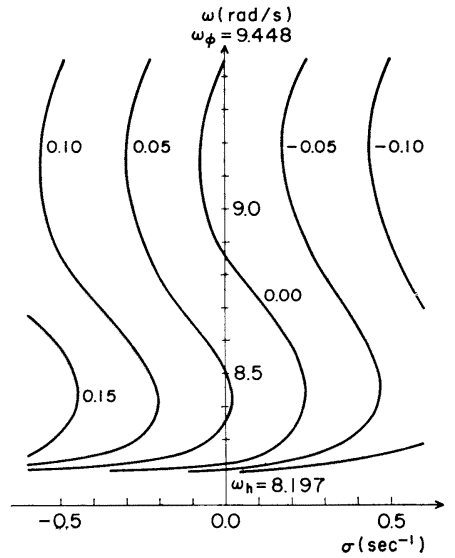

Bild 5-A $(\sigma, \omega)$-Darstellung der Stabilitätstheorie.

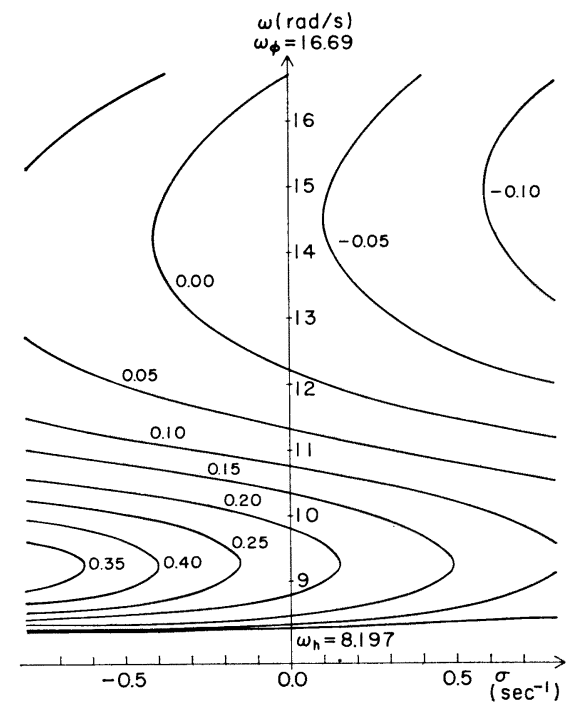

Bild 5-B $(\sigma, \omega)$-Darstellung der Stabilitätstheorie.
Erstens lassen wir das berechnete Resultat nach der Flatterntheorie ausdrücken. Es wird auf den Bildern 4-A und 4-B gezeigt. Sie werden $(U, g)$ Darstellung geheißt, weil ihre Querachse die Windgeschwindigkeit und ihre Längenachse die Strukturdämpfungskonstante sind. Gezeichneter Parameter ist die logarithmische Abnahme der unter dem dem Punkt $(U, g)$ entsprechenden Umstand existierenden Schwingung. Die Linie vom Parameter gleich Null entspricht dem Ergebnis der Flatterntheorie.

Zweitens lassen wir das Resultat, die Strukturdämpfungskonstante als den Parameter der Linien aufnehmend, gegen $\sigma$ und $\omega$ darstellen.

Wir lassen andere Beispiele, z.B. Darstellung gegen $U$ und $\sigma$, aus. Jedenfalls kann die Beziehung unter kleinen Veränderungen der Quantitäten genug genau geprüft werden dank der analytischen Form der Flatterngleichung.

\section{ANERKENNUNG}

Dieses Studium ist Prof. Dr. A. Hirai und Prof. Dr. M. Ito der Tokio Universität $z u$ besonderem Dank verpflichtet. Ohne ihr wohlwollendes Stützen und vor allem ohne ihren ausführlichen Rat kann es ja zweifellos nie ausgeführt worden sein.

Ich danke auch Prof. Dr. T. Inui der Chuo Universität für seine sorgfältige Anweisung zur numerischen Berechnung der in diesem Studium eine große Rolle spielenden Funktionen.

\section{LITERATUR}

1) 犬井鉄郎：特殊函数，岩波, S. 188 .

2) National Bureau of Standards: Columbia University Press

(1) Table of the Bessel Functions $J_{0}(z)$ and $J_{1}(z)$ for the complex Arguments.

(2) Table of the Bessel Functions $Y_{0}(z)$ and $Y_{1}(z)$ for the complex Arguments.

3) 熟津久一郎: 空力弾性学, 共立出版, S. 45 .

(Received Feb. 16, 1972) 